\title{
RVX-297, a BET Bromodomain Inhibitor, Has Therapeutic Effects in Preclinical Models of Acute Inflammation and Autoimmune Disease ${ }^{\mathbf{S}}$
}

\author{
Ravi Jahagirdar, Sarah Attwell, Suzana Marusic, Alison Bendele, Narmada Shenoy, \\ Kevin G. McLure, Dean Gilham, Karen Norek, Henrik C. Hansen, Raymond Yu, \\ Jennifer Tobin, Gregory S. Wagner, Peter R. Young, Norman C. W. Wong, \\ and Ewelina Kulikowski
}

Resverlogix Corp., Calgary, Alberta, Canada (R.J., S.A., K.G.M., D.G., K.N., H.C.H., R.Y., J.T., G.S.W., P.R.Y., N.C.W.W., E.K.); Hooke Laboratories Inc., Lawrence, Massachusetts (S.M.); Bolder BioPATH Inc., Boulder, Colorado (A.B.); and Aravasc Inc., Sunnyvale, California (N.S.)

Received August 24, 2017; accepted September 26, 2017

\begin{abstract}
Bromodomain (BD) and extra-terminal domain containing proteins (BET) are chromatin adapters that bind acetylated histone marks via two tandem BDs, BD1 and BD2, to regulate gene transcription. BET proteins are involved in transcriptional reprogramming in response to inflammatory stimuli. BET BD inhibitors (BETis) that are nonselective for BD1 or BD2 have recognized anti-inflammatory properties in vitro and counter pathology in models of inflammation or autoimmune disease. Although both $\mathrm{BD} 1$ and BD2 bind acetylated histone residues, they may independently regulate the expression of BET-sensitive genes. Here we characterized the ability of RVX-297, a novel orally active BETi with selectivity for BD2, to modulate inflammatory processes in vitro, in vivo, and ex vivo. RVX-297 suppressed inflammatory gene expression in multiple immune cell types in culture. Mechanistically, RVX-297 displaced BET proteins from
\end{abstract}

the promoters of sensitive genes and disrupted recruitment of active RNA polymerase II, a property shared with pan-BET is that nonselectively bind BET BDs. In the lipopolysaccharide model of inflammation, RVX-297 reduced proinflammatory mediators assessed in splenic gene expression and serum proteins. RVX-297 also countered pathology in three rodent models of polyarthritis: rat and mouse collagen-induced arthritis, and mouse collagen antibody-induced arthritis. Further, RVX-297 prevented murine experimental autoimmune encephalomyelitis (a model of human multiple sclerosis) disease development when administered prophylactically and reduced hallmarks of pathology when administered therapeutically. We show for the first time that a BD2-selective BETi maintains anti-inflammatory properties and is effective in preclinical models of acute inflammation and autoimmunity.

\section{Introduction}

Proinflammatory stimuli induce rapid transcriptional responses that are essential for host defense from infection. Regulation of inflammatory gene expression is coordinated

This research was funded by Resverlogix Corp. and did not receive any specific grant from funding agencies in the public, commercial, or not-for-profit sectors.

https://doi.org/10.1124/mol.117.110379.

S This article has supplemental material available at molpharm. aspetjournals.org. and tightly controlled to both elicit and retract the response. Although inflammation is critical for immune defense, the process is pathologic if exaggerated, chronically sustained, or directed against the individual's own tissue to generate an autoimmune condition. An inflammatory response involves a variety of cell types and the expression of inflammatory mediators such as cytokines and chemokines. Cytokines, including interleukin (IL)-6 and IL-1 $\beta$, have potent proinflammatory actions that contribute to the pathogenesis of autoimmune disorders (Choy and Panayi, 2001). IL-6 also stimulates differentiation of naive T cells into IL-17-producing

ABBREVIATIONS: ANOVA, analysis of variance; AUC, area under the curve; BD, bromodomain; BET, bromodomain and extra-terminal domain containing protein; BETi, bromodomain and extra-terminal domain containing protein inhibitor; BMDM, bone marrow-derived macrophage; BRD, bromodomain containing; CAS, clinical arthritis score; ChIP, chromatin immunoprecipitation; CIA, collagen-induced arthritis; CNS, central nervous system; $\mathrm{C}_{\mathrm{T}}$, threshold cycle; DMSO, dimethylsulfoxide; EAE, experimental autoimmune encephalomyelitis; FBS, fetal bovine serum; GM-CSF, granulocyte-macrophage colony-stimulating factor; IFN $\gamma$, interferon- $\gamma$; IL, interleukin; LPS, lipopolysaccharide; MAP, multianalyte profiling; mCAIA, mouse collagen antibody-induced arthritis; $\mathrm{mClA}$, mouse collagen-induced arthritis; MCP, monocyte chemotactic protein; MMP, matrix metalloproteinase; MOG, myelin oligodendrocyte glycoprotein; MS, multiple sclerosis; NF- $\kappa$ B, nuclear factor $\kappa$-light-chain-enhancer of activated B cells; NF- $\kappa$ Bia, nuclear factor- $\kappa$-light-chain-enhancer of activated B cells inhibitor $\alpha$; OKT3, anti-CD3 antibody; PBMC, peripheral blood mononuclear cell; PBS, phosphate-buffered saline; PCR, polymerase chain reaction; PMA, phorbol 12-myristate 13-acetate; P-Ser2, phosphorylated on serine 2; RA, rheumatoid arthritis; RANKL, receptor activator of nuclear factor $\kappa-B$ ligand; rCIA, rat collagen-induced arthritis; RNA pollI, RNA polymerase II; $\mathrm{T}_{\mathrm{H}}$, T helper; TNF $\alpha$, tumor necrosis factor $\alpha$; VCAM-1, vascular cell adhesion molecule 1. 
T-helper cells $\left(\mathrm{T}_{\mathrm{H}} 17\right.$ cells) that promote amplification of the inflammatory response in a feed forward mechanism (Dienz and Rincon, 2009). $\mathrm{T}_{\mathrm{H}} 17$ cells play a critical role in the development of autoimmune disorders, including rheumatoid arthritis (RA) and multiple sclerosis (MS). In autoimmune disorders, IL-17 mediates tissue inflammation through signal transduction that recruits and activates neutrophils and macrophages, and promotes the expression of proinflammatory chemokines and cytokines (Ye et al., 2001; Kolls and Linden, 2004). Current evidence suggests that IL-17 plays an active role in inflammatory diseases, autoimmune disorders, and cancer (Kolls and Linden, 2004).

Extensive transcriptional reprogramming is invoked by proinflammatory stimuli (Brown et al., 2014). Epigenetic modifications to chromatin provide a framework for the rapid alterations in gene expression required for immune cell activation and differentiation (Wei et al., 2009). Epigenetics regulate gene expression through covalent modifications of chromatin that impact chromatin structure and the recruitment of transcriptional complexes. Histone acetylation is one such modification associated with active gene transcription. Acetylation occurs on lysine residues, which are recognized by chromatin readers such as the bromodomain (BD) and extraterminal domain containing protein (BET) family of proteins (BRD2, BRD3, BRD4, and the testis-specific BRDT). BET proteins contain two tandem BDs, BD1 and BD2, that interact with acetylated lysines on histone tails and recruit transcriptional machinery to regulate gene expression (Filippakopoulos et al., 2012). Pharmacological BET inhibitors (BETi) that bind BD1 and BD2 with similar affinity (pan-BETi) can suppress inflammatory gene expression; they prevent BET protein associations with acetylated histones at the promoters of genes encoding inflammatory cytokines (Nicodeme et al., 2010; Bandukwala et al., 2012; Brown et al., 2014). Both genetic approaches and pan-BETi studies have demonstrated that BET proteins are critical for inflammatory responses in macrophages, including the expression of IL-6 and other key inducible cytokines and chemokines (Nicodeme et al., 2010). Roles for BET proteins in both acute inflammatory responses (Nicodeme et al., 2010; Belkina et al., 2013) as well as autoimmune pathology involving $\mathrm{T}_{\mathrm{H}} 17$ cell differentiation (Mele et al., 2013) have been established. BET proteins are involved not only in the differentiation of naive $\mathrm{CD}^{+} \mathrm{T}$ cells from $\mathrm{T}_{\mathrm{H}} 17$ cells, but also in the activation of previously differentiated $\mathrm{T}_{\mathrm{H}} 17$ cells (Mele et al., 2013). Further, $\mathrm{T}$ cellmediated immunity involves the maturation of dendritic cells, which have also been linked to autoimmune disease (Baumgart and Carding, 2007). Dendritic cells play a stabilizing role in the immune response and induce T-cell immunity upon maturation, whereas immature cells promote immune tolerance. A pan-BETi can impair full maturation of monocyte-derived dendritic cells, resulting in decreased induction of proinflammatory T cells (Toniolo et al., 2015). Hence, several lines of evidence indicate that BETis have a strong therapeutic potential to suppress the inflammatory processes that underlie autoimmune disorders.

Studies have shown that BD selective BETis modify different transcriptional outcomes compared with non-selective BETis (Picaud et al., 2013; Gacias et al., 2014), implying that BD selectivity may have differential biological consequences that impact inflammation. RVX-297 is a novel, orally bioavailable BETi that has a 47- to 58-fold greater affinity for BD2 than for BD1 in the BET family of proteins (Kharenko et al., 2016). In this report, we characterize the anti-inflammatory properties of RVX-297 and demonstrate for the first time that a BD2-selective BETi impacts gene expression and cellular function in immune cell types that are involved in inflammation and autoimmunity, including macrophages, $\mathrm{T}$ cells, $B$ cells, and synovial fibroblasts. We also investigate the efficacy of RVX-297 in preclinical models of endotoxemia, polyarthritis (Bendele, 2001), and T cell-mediated autoimmune disease with features of MS (Thakker et al., 2007). Our results demonstrate that $\mathrm{BD} 2$-selective inhibition of $\mathrm{BET}$ proteins has anti-inflammatory properties in vitro and in animal models of autoimmune disease. Based on these data, we suggest that selective inhibition of BD2 has potential in ameliorating autoimmune disorders by simultaneously modulating inflammatory factors in multiple cell types.

\section{Materials and Methods}

\section{Materials}

I-BET762, JQ1, and RVX-297 were synthesized as previously described (Filippakopoulos et al., 2010; Nicodeme et al., 2010; Hansen, 2011). Dexamethasone and FTY720 were obtained from commercial sources.

\section{U937 Cell Treatment}

U937 cells (ATCC, Manassas, VA) were differentiated to macrophage-like cells with $60 \mathrm{ng} / \mathrm{ml}$ phorbol 12-myristate 13 -acetate (PMA) for 3 days. Cells were pretreated for 1 hour with RVX-297 or dimethylsulfoxide (DMSO) without PMA prior to the addition of LPS $(1 \mu \mathrm{g} / \mathrm{ml})$. Cells were incubated for 3 hours before gene expression analysis by real-time polymerase chain reaction (PCR). Each data point was derived from three biological replicates, and results were determined in three independent experiments.

\section{Primary B-Cell Isolation and Treatment}

Spleens from C57BL/6 mice were rinsed in cold phosphate-buffered saline (PBS) and disrupted under a syringe plunger. The cell suspension was passed through a 20 -gauge syringe three times and filtered through a $50-\mu \mathrm{m}$ filcom. Cells were pelleted by centrifugation then resuspended in a red blood cell lysis solution. Cells were pelleted again and resuspended in an isolation buffer $[\mathrm{Ca} / \mathrm{Mg}$-free PBS with $2 \mathrm{mM}$ EDTA and $2 \%$ fetal bovine serum (FBS)]. B cells were isolated using mouse CD43 beads (Life Technologies, Carlsbad, CA) according to the manufacturer's instructions. Cells were plated at 15,000 cells/well in 96-well format in RPMI medium containing 10\% FBS and treated with RVX-297 or DMSO, LPS $(1 \mu \mathrm{g} / \mathrm{ml})$, and ionomycin (1 $\mu \mathrm{M})$. Incubation was maintained for 3 hours followed by gene expression analysis. Results were verified in an independent experiment.

\section{IL-17 Expression}

IL-17 gene expression was analyzed in human peripheral blood mononuclear cells (PBMCs). Briefly, PBMCs (Cellular Technologies Ltd., Shaker Heights, OH) were thawed in OpTmizer T-cell expansion media (Life Technologies) supplemented with $20 \mathrm{ng} / \mathrm{ml}$ IL-2 (Sigma-Aldrich, St. Louis, MO). Cells were pelleted by centrifugation and resuspended in media recommended by the supplier. Cells were pretreated with RVX-297 or DMSO for 1 hour, then media containing anti-CD3 antibody (OKT3; eBioscience, San Diego, CA) was added at a final concentration of $1 \mu \mathrm{g} / \mathrm{ml}$. Incubation was continued for 3 hours. IL-17 gene expression was analyzed by real-time PCR. Each data point was derived from three biological replicates, and results were determined in six independent experiments. 


\section{Monocyte Chemotactic Protein 1 Assay}

Cryopreserved PBMCs (Cellular Technologies Ltd.) were plated in 96-well format in RPMI media (Life Technologies) containing 10\% FBS. Cells were treated for 3 hours with each condition in triplicate before harvesting for gene expression analysis by real-time PCR. RVX-297 mediated repression of monocyte chemotactic protein (MCP)-1 was verified in four independent experiments as well as in PBMCs from three different donors.

\section{Mouse Bone Marrow-Derived Macrophage Isolation and Treatment}

Primary bone marrow-derived macrophages (BMDMs) were isolated from mouse femurs. Briefly, femurs from 6-week-old C57BL/6 mice were cut with a scalpel at both ends and flushed with RPMI media. Cells were washed and resuspended in media supplemented with $20 \% \mathrm{FBS}, 40 \mathrm{ng} / \mathrm{ml} \mathrm{GM}-\mathrm{CSF}$, and $5 \mathrm{ng} / \mathrm{ml} \mathrm{IL-3,} \mathrm{then} \mathrm{were} \mathrm{allowed}$ to differentiate for 6 days. Cells were replated in a 24 -well format $(200,000$ cells/well) for further treatments. Each treatment condition was performed in triplicate. Expression of IL- 6 and IL- $1 \beta$ were analyzed by real-time PCR. Results were verified in an independent experiment.

\section{Human Rheumatoid Arthritis Synovial Fibroblasts}

Synovial fibroblasts isolated from the knee of arthritic patients (Asterand Bioscience, Detroit, MI) were plated at 6000 cells/well in 96-well format in Dulbecco's modified Eagle's medium containing 10\% FBS. Fibroblasts were treated identically to U937 cells, except stimulation was induced with $30 \mathrm{ng} / \mathrm{ml} \mathrm{TNF} \alpha$. Real-time PCR was performed after 24 hours of treatment. Representative data involving three biological replicates per treatment group are shown. The experiment was performed four times, and results were verified in cells derived from a different donor.

\section{Real-Time PCR}

mRNA was isolated from cell culture systems using Catcher Plus kits (Life Technologies) or from tissue samples using TRIzol (Life Technologies) according to the manufacturer's instructions. Gene expression was measured by TaqMan-based real-time PCR using the RNA UltraSense One-Step qRT-PCR System, as described previously (McLure et al., 2013; Gilham et al., 2016). mRNA levels were measured relative to an endogenous control on 7500 or ViiA-7 Real Time PCR Instruments (Applied Biosystems, Foster City, CA).

\section{Chromatin Immunoprecipitation}

BMDMs were plated in $10 \mathrm{~cm}$ dishes $6 \times 10^{6}$ cells/plate. After 1 day, cells were treated with compound for 1 hour; LPS $(1 \mu \mathrm{g} / \mathrm{ml}$ final concentration) and RVX-297 $(10 \mu \mathrm{M})$ or DMSO $(0.1 \%)$ were added, and incubations were continued for 3 hours. Chromatin immunoprecipitation (ChIP) was performed essentially as previously published (McCarthy et al., 2003; Nicodeme et al., 2010). Briefly, cells were crosslinked with $1 \%$ formaldehyde for 10 minutes before quenching with glycine. Chromatin was sheared by sonication, and insoluble debris was removed by centrifugation. Immunoprecipitation was performed with $500 \mu \mathrm{g}$ of protein and $2 \mu \mathrm{g}$ of BRD antibodies (BRD2 a302-583a, BRD3 a302-368a, and BRD4 a301-985a; Bethyl Laboratories, Montgomery, TX), RNA polymerase II (polII; ab5095; Abcam), tetra-acetylated Histone H4 (06-866; Millipore, Billerica, MA), or IgG (I5006; Sigma-Aldrich). A corresponding input sample was treated equally. After de-crosslinking, DNA was isolated by phenol chloroform extraction, and real-time PCR was performed using Power SYBR Green reagent for detection (Applied Biosystems). PCR primer sequences were published by Nicodeme et al. (2010), and the fold difference was calculated as $2^{\wedge}\left[\mathrm{C}_{\mathrm{T}}\right.$ (input) $\left.-\mathrm{C}_{\mathrm{T}}(\mathrm{ChIP})\right]$.

\section{Animal Studies}

All animal studies adhered to standard operating procedures, received animal ethics approval from the Animal Welfare Committee under institutional animal care and use committee guidelines, and conformed to good scientific practice.

\section{Compound Formulation in Animal Studies}

RVX-297 was dosed orally for all animal studies and was prepared in formulation EA006 as described by Jahagirdar et al. (2014). The concentration of the test compound in each preparation was verified by liquid chromatography tandem mass spectrometry.

\section{LPS-Induced Endotoxemic Mouse Model}

Endotoxemia studies in mice were performed at Aravasc (Sunnyvale, CA). Eight-week-old male C57BL/6 mice (Charles River, Worcester, MA, or Harlan Laboratories, Indianapolis, IN) received LPS (Sigma-Aldrich) by intraperitoneal injection. RVX-297 was administered orally at $75 \mathrm{mg} / \mathrm{kg} 4$ hours before and again at the time of stimulation with an amount of LPS producing an optimal response. For serum cytokine determinations, animals received $5 \mu \mathrm{g}$ of LPS. Serum levels of IL-6, IL-17, and interferon- $\gamma($ IFN $\gamma)$ were determined by enzyme-linked immunosorbent assay (R\&D Systems Inc., Minneapolis, MN) 4 hours after LPS administration. Multianalyte profiling (MAP) of serum was performed as outlined in the Supplemental Material. Changes in gene expression, which precede changes in circulating protein levels, were measured in the spleen by real-time PCR as above except measurements were taken 4 hours after receiving $10 \mu \mathrm{g}$ of LPS.

\section{Rat Collagen-Induced Arthritis}

Rat collagen-induced arthritis (rCIA) studies were performed at Aravasc. Female Lewis rats (Charles River) were 6-8 weeks old and weighed approximately $150 \mathrm{~g}$. Bovine type II collagen (Chondrex Inc., Redmond, WA) was emulsified with incomplete Freund's adjuvant. Rats were immunized by intradermal injection in the sacrolumbar region and at the base of the tail, and a booster was provided on day 7 . Beginning on day 11 (arthritis phase), RVX-297 was administered orally twice per day for 6 days at 25, 50 , and $75 \mathrm{mg} / \mathrm{kg} /$ dose. Body weight was tracked, and ankle diameter was monitored via caliper measurements. Systemic plasma exposure of RVX-297 was determined 1 day prior to the end of the study for pharmacokinetic/pharmacodynamic analysis (see Supplemental Material). Histopathology of the ankle and knee (in the right paws; see Supplemental Material), gene expression, and cytokine levels in the ankle (left paws) were evaluated at the end of the study.

\section{Mouse Collagen-Induced Arthritis}

See Supplemental Material.

\section{Mouse Collagen Antibody-Induced Arthritis}

Mouse collagen antibody-induced arthritis (mCAIA) studies were performed at Aravasc and conducted as previously described (Khachigian, 2006). Briefly, female BALB/cAnNHsd mice (Harlan Laboratories) weighing approximately $18 \mathrm{~g}$ were injected intravenously with $2.5 \mathrm{mg} /$ mouse cocktail containing four arthritogenic monoclonal antibodies to collagen-II (MD Bioproducts, St. Paul, $\mathrm{MN}$ ). After 24 hours, mice received $25 \mu \mathrm{g}$ of LPS (Sigma-Aldrich) by intraperitoneal injection to enhance the inflammatory response. Arthritis symptoms developed 24-48 hours later. Twice per day oral administration of RVX-297 (150 mg/kg) or dexamethasone, an antiinflammatory corticosteroid $(0.5 \mathrm{mg} / \mathrm{kg})$, was initiated 30 hours after LPS administration and continued for 9 days during the arthritis phase. Efficacy evaluation was based on body weight, clinical arthritis scores (CASs) (all four paws), combined hind paw weights, and 
histologic assessment of fore paws, hind paws, and knees (see Supplemental Material).

\section{Murine Experimental Autoimmune Encephalomyelitis}

Experimental autoimmune encephalomyelitis (EAE) studies were performed as outlined previously (Thakker et al., 2007; Bittner et al., 2014) at Hooke Laboratories Inc. (Lawrence, MA). This model of autoimmunity was used in both therapeutic and preventative regimens. EAE was induced in female C57BL/6 mice weighing 18-23 g (Taconic Farms, Hudson, NY) by immunization with myelin oligodendrocyte glycoprotein (MOG) peptides 35-55 in complete Freund's adjuvant. Two hours and 24 hours after immunization, pertussis toxin was administered by intraperitoneal injection. Compounds were delivered by oral gavage at 75 and $125 \mathrm{mg} / \mathrm{kg}$ BID for RVX-297 or $0.5 \mathrm{mg} / \mathrm{kg}$ QD for FTY720 for 18 days once disease was established (i.e., at the first sign of EAE) in a therapeutic regimen, or for 27 days immediately after MOG immunization in a preventative regimen. Given preventatively, RVX-297 was not well tolerated at $125 \mathrm{mg} / \mathrm{kg}$, and was adjusted to $100 \mathrm{mg} / \mathrm{kg}$ BID on day 18 of treatment. Vehicle and RVX-297 $75 \mathrm{mg} / \mathrm{kg}$ treatment cohorts were composed of 10 mice, whereas the RVX-297 $125 \mathrm{mg} / \mathrm{kg}$ treatment cohort had 9 mice. EAE development and clinical score were evaluated based on clinical manifestations published by Bittner et al. (2014). The mean maximum clinical score and body weight were tracked. Spinal cords were collected at the end of the treatment period for gene expression analysis and examination of infiltrating cells by flow cytometry, as outlined below. Histological assessment of inflammatory foci, apoptotic cells, and demyelination in the cervical, thoracic, and lumbar regions of the spinal cord were performed by Hooke Laboratories (Lawrence, MA; hookelabs.com) using the protocol of Marusic et al. (2008) by an experienced observer blinded to the treatment group.

\section{Ex Vivo Assessment of EAE Mice}

Lymphocytes. Lymphocytes isolated from inguinal and axilar nodes of EAE mice that had been treated with vehicle or RVX-297 for 11 days were cultured for 72 hours in the presence of MOG peptides $35-55$. The levels of secreted cytokines in cell supernatants were determined through cytometric bead assays.

CNS Infiltrating Cells. After perfusion with PBS, cells from brain and spinal cord tissue were isolated by density gradient centrifugation. Isolated cells were cultured with PMA (50 ng/ml), ionomycin $(0.5 \mu \mathrm{g} / \mathrm{ml})$, and brefeldin $(1 \mu \mathrm{g} / \mathrm{ml})$ for $4-5$ hours before analysis of the cells by flow cytometry, as previously described (Bandukwala et al., 2011).

\section{Statistical Analysis}

Student's $t$ test (two-tailed, unpaired) was used to evaluate differences between control groups and a single-test treatment group. When multiple test treatments were used, a one-way analysis of variance (ANOVA) followed by Tukey's multiple-comparison test was used. Variance is shown as the S.D. in figures and tables.

\section{Results}

RVX-297 Decreases Proinflammatory Gene Expression in Multiple Cell Types In Vitro. The effect of RVX-297 on gene expression was evaluated in several immune cell types involved in autoimmunity and inflammation. IL-6 is an acute-phase inflammatory cytokine that is secreted by a variety of cell types including macrophages, $\mathrm{T}$ cells, $\mathrm{B}$ cells, and fibroblasts; its transcription and secretion are induced rapidly after stimulation with LPS or IL- $1 \beta$. RVX-297 suppressed IL-6 gene induction in human U937 macrophages, mouse primary B cells isolated from the spleen, mouse BMDMs (Table 1), and THP-1 monocytes (data not shown) in
TABLE 1

RVX-297 reduces gene expression of inflammatory mediators in vitro Cells were treated with inflammatory stimuli and RVX-297 as described in Materials and Methods. Each data point in the dose-response analysis was collected using biological triplicates. Gene expression was measured by real-time PCR, and $\mathrm{IC}_{50}$ values were determined through nonlinear regression (normalized response and variable slope) using GraphPad Prism software version 5.04. Results are shown as the mean \pm S.D. when replicated in at least three independent experiments or as the range from two independent experiments.

\begin{tabular}{lllc}
\hline mRNA & \multicolumn{1}{c}{ Cell System } & \multicolumn{1}{c}{ Stimulant } & $\begin{array}{c}\mathrm{RVX}-297 \mathrm{IC}_{50} \\
(\mu \mathrm{M})\end{array}$ \\
\hline IL-6 & Human U937 & LPS & $0.71 \pm 0.07$ \\
MCP-1 & Human PBMC & None & $0.4 \pm 0.2$ \\
IL-17 & Human PBMC & OKT3 & $1.3 \pm 0.5$ \\
IL-6 & Mouse B cells & LPS and ionomycin & $0.46-0.53$ \\
IL-6 & Mouse BMDM & LPS & $0.4-3.0$ \\
IL-1 $\beta$ & Mouse BMDM & LPS & $2.1-23.6$ \\
\hline
\end{tabular}

a dose-dependent manner. IL- $1 \beta$ is another proinflammatory cytokine linked to autoimmune disorders that is expressed in activated monocytes and macrophages (Dinarello, 2009). In LPS-stimulated mouse BMDMs, IL- $1 \beta$ expression was repressed by RVX-297, demonstrating suppressive effects on multiple inflammatory mediators (Table 1). A downstream target of IL-6, MCP-1, was examined in human PBMCs. MCP-1 is a chemokine that regulates the migration and infiltration of monocytes and macrophages at sites of injury or infection (Carr et al., 1994). Although MCP-1 gene expression is potently induced by IL-6 (Biswas et al., 1998), RVX-297 inhibited MCP-1 expression in unstimulated human PBMCs (Table 1). This suggests that RVX-297 downregulates the expression of inflammatory mediators independent from suppression of IL-6. PBMCs were also used as a source of T cells. When PBMCs were stimulated with the T-cell receptor antibody OKT3, IL-17 mRNA was induced, indicating the activation of $\mathrm{T}_{\mathrm{H}} 17$ cells. RVX-297 inhibited antigen stimulation of T cells and the induction of IL-17 expression (Table 1). IL-6 and IL-17 are critical factors in the progression of autoimmunity, and the downregulation of these cytokines suggests that BETi can impact multiple processes contributing to inflammation.

RVX-297 Decreases Proinflammatory Gene Expression in Synovial Fibroblasts Isolated from Patients with Rheumatoid Arthritis. RVX-297 treatment of synovial fibroblasts, isolated from the knee fluid of patients with RA, repressed inflammatory gene expression. Resident synovial fibroblasts contribute significantly to the perpetuation of RA and may even play a role in its initiation (Lefevre et al., 2015). TNF $\alpha$ treatment induced IL-6 gene expression in human synovial fibroblasts, an effect countered by RVX-297 in a dose-dependent manner (Fig. 1A). Consistent with our result, the pan-BETi JQ1 and BRD4 small interfering RNAs have both been reported to suppress IL-6 protein secretion from $\mathrm{TNF} \alpha$-stimulated human synovial fibroblasts (Zhang et al., 2015). Similarly, the expression of another inflammatory marker, vascular cell adhesion protein 1 (VCAM-1), was also induced with $\mathrm{TNF} \alpha$ treatment, which was countered by RVX-297 administration (Fig. 1B). These data show that RVX-297 can modulate the inflammatory response of resident synovial fibroblasts, whose activity is dysregulated in RA.

Mechanism of BETi Suppression of Proinflammatory Gene Expression. RVX-297 and pan-BETis (JQ1 and I-BET762) were found to inhibit inflammation-induced gene expression in primary mouse BMDMs (Fig. 2A). LPS stimulation induced IL-6 gene expression as expected (Nicodeme et al., 2010), 

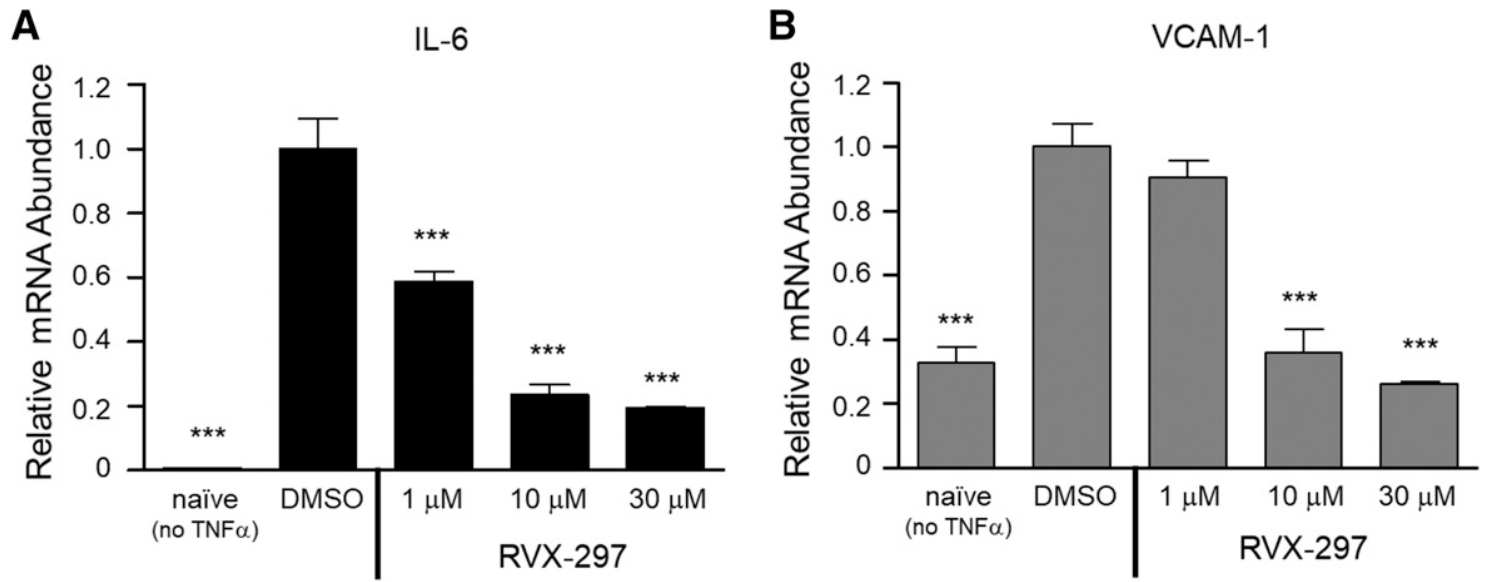

Fig. 1. RVX-297 downregulates IL-6 and VCAM-1 gene expression in synovial fibroblasts from patients with rheumatoid arthritis. Synovial fibroblasts isolated from fluid obtained from patients with RA were stimulated with TNF $\alpha$ and treated with RVX-297 for 24 hours. Gene expression was measured by real-time PCR for IL-6 (A) or VCAM-1 (B). Data are the mean \pm S.D. of independent triplicate samples. One-way ANOVA followed by Tukey's multiplecomparison test was used to determine statistical significance compared with DMSO-treated cells $(* * * P<0.001)$.

and this induction was inhibited by RVX-297 in a dose-dependent manner (Fig. 2A; Table 1). Both JQ1 (Filippakopoulos et al., 2010) and I-BET762 (Nicodeme et al., 2010) pan-BETis, with chemical scaffolds distinct from RVX-297, also inhibited LPSinduced IL- 6 gene expression. The upregulation of IL-1 $\beta$ and
$\mathrm{TNF} \alpha$ gene expression was also opposed by all three BETi compounds (RVX-297, JQ1, and I-BET762; data not shown). Thus, the induction of proinflammatory cytokines IL- 6 , IL- $1 \beta$, and $\mathrm{TNF} \alpha$ can be blocked by pharmacological inhibition of BET BDs, the mutual targets of BETis with different chemical scaffolds.

B
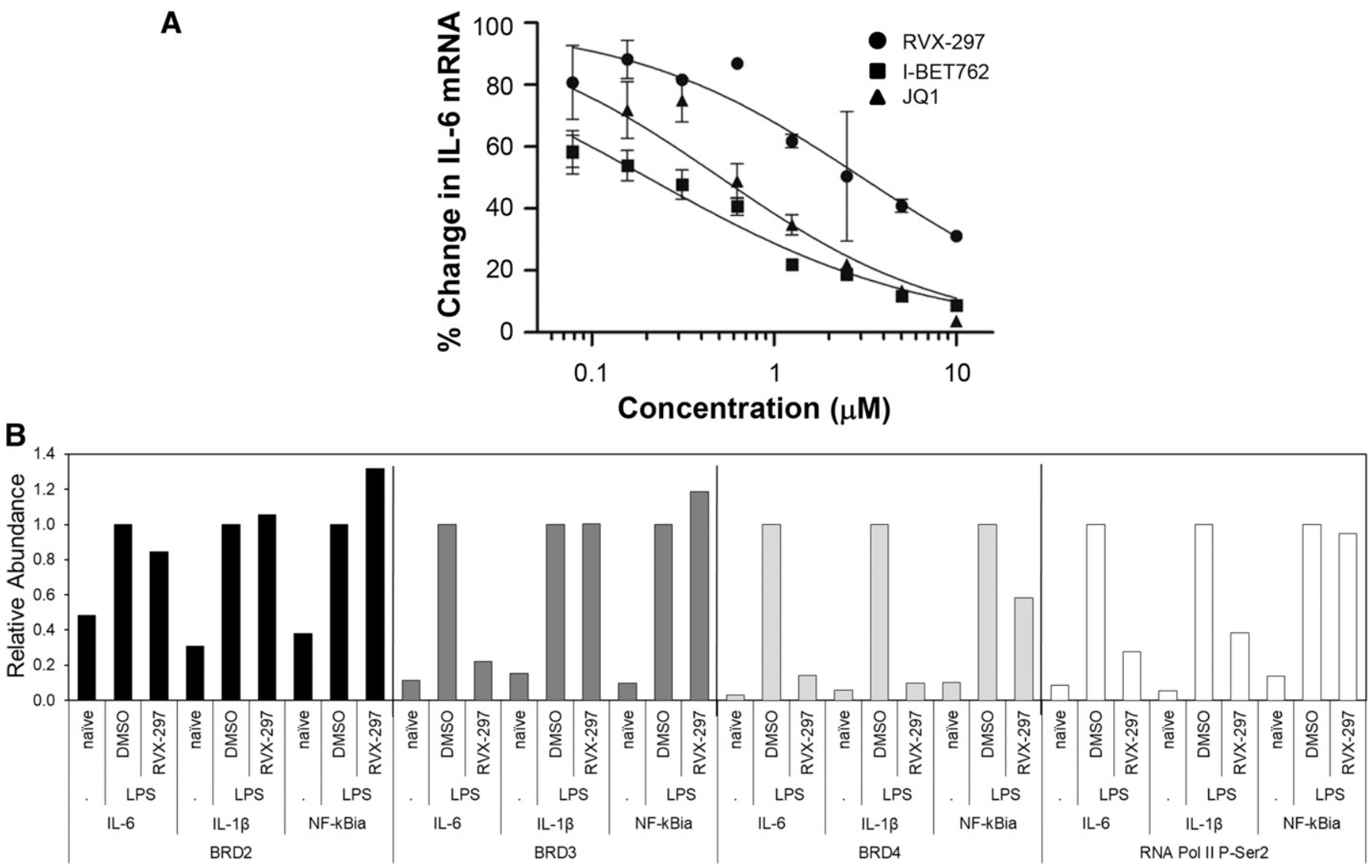

Fig. 2. BET bromodomains regulate macrophage cytokine gene expression. (A) Mouse BMDMs were stimulated with LPS and then treated with different concentrations of RVX-297 or pan-BETi with distinct chemical scaffolds (I-BET762 and JQ1) for 3 hours. Gene expression was assessed by realtime PCR. Each data point represents the mean \pm S.D. of independent triplicate samples. IC $_{50}$ values were 3.0, 0.2, and 0.5 $\mu \mathrm{M}$ for RVX-297, I-BET762, and JQ1 respectively. (B) Mouse BMDMs were stimulated with LPS and treated with $10 \mu \mathrm{M}$ RVX-297 or 0.1\% DMSO. ChIP was performed for BRD2, 3, and 4 or active RNA polII P-Ser2, and their relative abundance on the promoter of the genes indicated was determined. IgG was used as a negative control. Data are representative of two independent experiments. 
ChIP studies revealed that RVX-297 interferes with BET protein binding to inflammatory gene promoters. As shown in Fig. 2B, LPS treatment led to increased association of BRD2, 3, and 4 at the promoters of the BETi-sensitive genes IL- 6 and IL-1 $\beta$ (genes downregulated by BETi) and the $\mathrm{BETi}$-insensitive gene NF- $\kappa \mathrm{Bia}$ (nuclear factor- $\kappa$-light-chainenhancer of activated B cells inhibitor $\alpha$ ) (Nicodeme et al., 2010). RVX-297 reduced the binding of BRD3 and BRD4, but not BRD2, to the IL-6 promoter (Fig. 2B), which is in agreement with the reduced expression of the corresponding mRNA (Table 1). RVX-297 also reduced the abundance of BRD4 on the IL- $1 \beta$ promoter, which again is in line with BETisensitive gene expression (Table 1). However, BET protein abundance on the NF- $\kappa$ Bia promoter was unaffected by RVX-297 BETi treatment, which is consistent with its reported insensitivity to BETi (Nicodeme et al., 2010). Site-specific alterations in BET protein abundance induced by BETi imply that the results of the ChIP assays were not a consequence of globally altered chromatin structure.

$\mathrm{BET}$ proteins, in particular BRD4, have been reported to regulate the activity of RNA polII through the recruitment of positive transcription elongation factor $b$ to activate transcription (Yang et al., 2005; Itzen et al., 2014). The active form of RNA polII is phosphorylated on serine 2 (P-Ser2), and its abundance on a gene is related to levels of transcription (for review, see Phatnani and Greenleaf, 2006). RVX-297 treatment reduced the amount of RNA polII P-Ser2 on the IL- 6 and IL- $1 \beta$ promoters, but not NF- $\kappa$ Bia (Fig. $2 \mathrm{~B}$ ), which is consistent with reduced transcription of IL- 6 and IL-1 $\beta$ (Table 1). There was no change in the level of acetylated histone H4 on the IL- 6 promoter (data not shown), suggesting that BETi promotes the displacement of BET proteins from chromatin without altering the associated histone acetylation. Cumulatively, our results indicate that the RVX-297mediated reduction in IL- 6 and IL- $1 \beta$ gene expression can be explained by the displacement of BET proteins from acetylated histones, which disrupts the recruitment of transcriptionally active RNA polII to the promoter of BET-sensitive genes. In this system, RVX-297 effects on the abundance of BRD3 and 4 on the gene promoter were more predictive of transcription levels than was the abundance of BRD2.

A

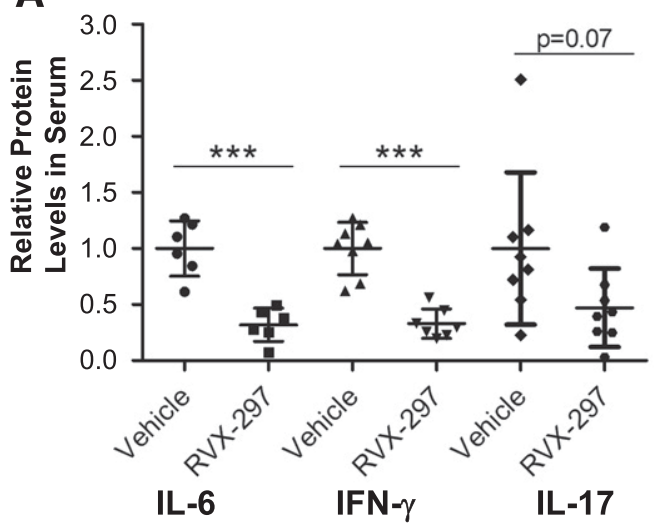

RVX-297 Suppresses Cytokine Production in LPSTreated Mice. Endotoxin injection has been widely used to study the acute inflammatory response; here we show that RVX-297 delivered 3-4 hours before stimulation, and again at the time of stimulation, reduced both cytokine production and gene expression in LPS-stimulated endotoxemic mice. Circulating levels of LPS-induced IL-6, IL-17, and IFN $\gamma$ were lower in the serum of RVX-297-treated mice compared with vehicle-treated controls (Fig. 3A). In concordance, IL-6 and IL-17 gene expression measured in the spleen isolated from LPS-stimulated endotoxemic mice was also decreased (Fig. 3B). MAP analysis of serum from LPS-stimulated endotoxemic mice demonstrated lower levels of numerous circulating cytokines, chemokines, and adhesion molecules after RVX-297 treatment, including GM-CSF, MCP-1, MCP-5, IL-2, $\mathrm{TNF} \alpha$, and regulated on activation normal $\mathrm{T}$ cell-expressed and secreted (RANTES) (Supplemental Table 1). These results confirm that RVX-297 has broad and rapid anti-inflammatory effects in a mouse model of endotoxemia.

RVX-297 Inhibits Progression of Pathology in the Rat Collagen-Induced Arthritis Model. Analysis of antiinflammatory properties of RVX-297 was extended into animal models of autoimmunity. Collagen-induced arthritis (CIA) is a commonly used rodent model of human RA (Williams, 2004). In the rCIA model, collagen injections caused a significant increase in rat ankle diameter that developed over time in vehicle-treated arthritic animals as expected (Fig. 4A). This increase in ankle diameter was attenuated by RVX-297 treatment, with differences apparent at day 2 for 50 and $75 \mathrm{mg} / \mathrm{kg}$ RVX-297 (ANOVA with Tukey's test, $P<0.05$ ), and at all doses of RVX-297 in the following days (ANOVA with Tukey's test, $P<0.01$ for $25 \mathrm{mg} / \mathrm{kg}$ and $P<0.001$ for 50 and $75 \mathrm{mg} / \mathrm{kg}$ doses) (Fig. 4A). In fact, ankle diameter did not differ significantly between animals in the RVX-297 treatment groups and naive animals. RVX-297 also dramatically reduced synovitis, cartilage damage, pannus formation, and bone resorption in the ankle and knee joint, which was readily observed in toluidine blue-stained sections (Fig. 4B). Pharmacokinetic/pharmacodynamic analysis of ankle diameter and total ankle or knee histopathology showed

B

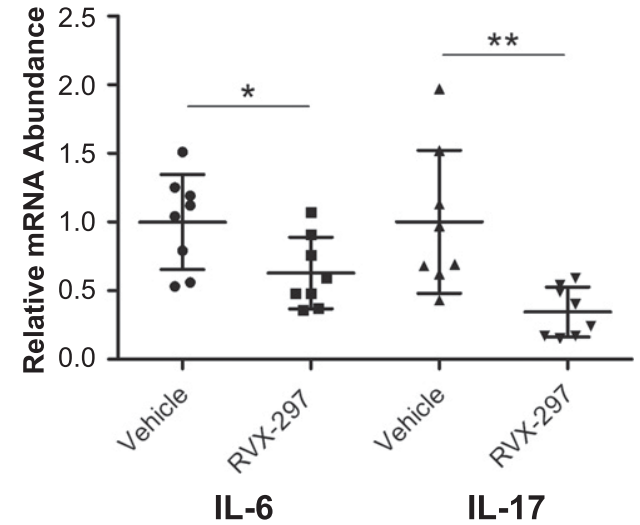

Fig. 3. RVX-297 suppresses LPS-induced cytokine production in mice. RVX-297 or vehicle was delivered to C57BL/6 mice 4 hours before stimulation and again when stimulated with LPS. (A) Serum cytokine levels were determined by enzyme-linked immunosorbent assay (ELISA) 4 hours after LPS administration. (B) Gene expression in the spleen was determined by real-time PCR 4 hours post-LPS. $n=6-8$ animals/group. Graphs show the mean \pm S.D. Statistical significance was determined with a Student's $t$ test $(* P<0.05 ; * * P<0.01 ; * * P<0.001)$. 
A

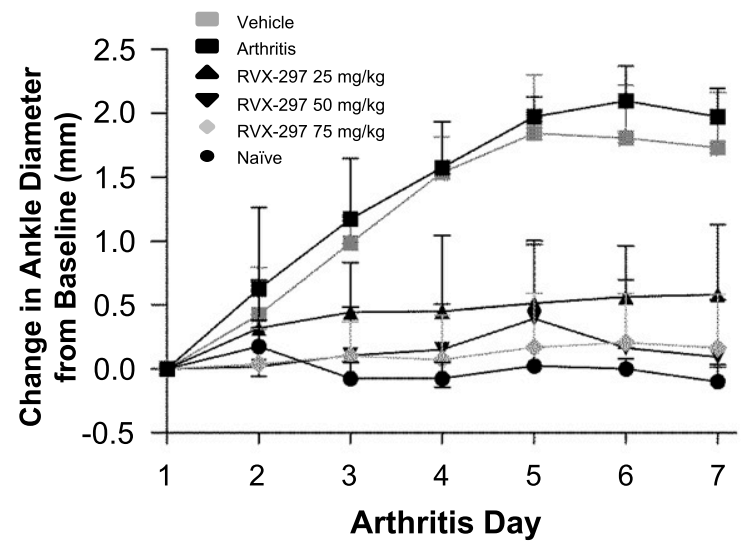

B
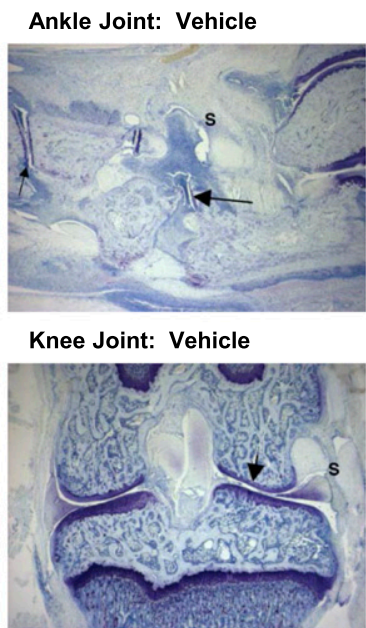

RVX-297 $75 \mathrm{mg} / \mathrm{kg}$

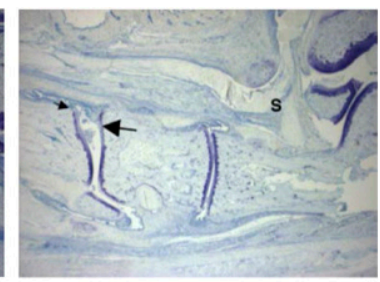

RVX-297 75 mg/kg

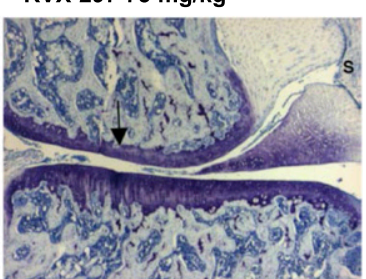

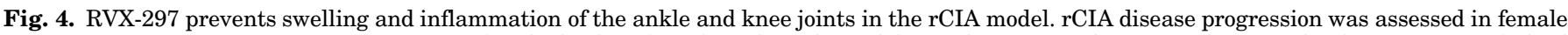

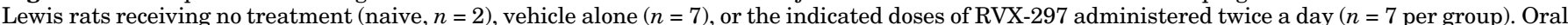

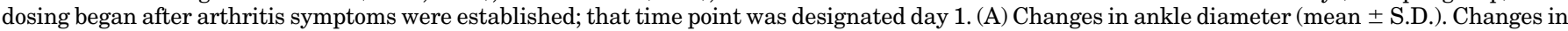

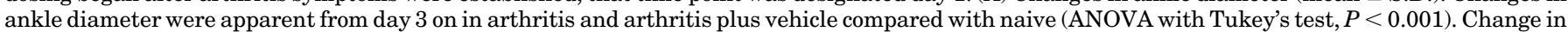

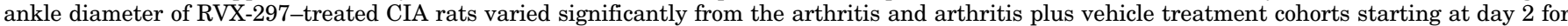

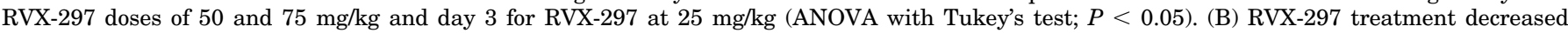

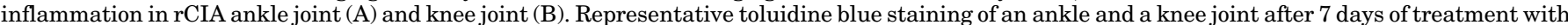

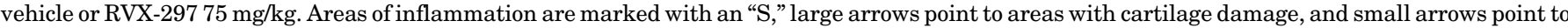
areas of pannus formation.

that effects of RVX-297 reached a maximum at approximately $50 \mathrm{mg} / \mathrm{kg}$ BID (Table 2). This near-maximal pharmacodynamic response corresponds to a RVX-297 systemic exposure [area under the curve (AUC) 0-12 hours] of approximately $50,000 \mathrm{~h} * \mathrm{ng} / \mathrm{ml}$, which produced a clear and pronounced antiinflammatory effect on all of the efficacy parameters assessed.

In the rCIA model, RVX-297 treatment also had a substantial impact on cytokine gene expression; expressions of IL-1 $\beta$, RANKL, MMP3, and MMP13 were all significantly decreased in the ankle joints of arthritic rats after RVX-297 treatment at $75 \mathrm{mg} / \mathrm{kg}$ BID versus vehicle-treated arthritic controls (Table 3; Student's $t$ test). In addition, IL-6 and VCAM-1 protein levels were significantly lower in the ankles of arthritic rats treated with RVX-297 versus vehicle (Table 3; Student's $t$ test). Further, MAP analysis demonstrated that additional protein markers of inflammation were also reduced in the ankle joint of RVX-297-treated animals (data not shown). Together, RVX-297 BETis had suppressive effects on the production of cytokines, adhesion molecules, and inflammatory factors, culminating in substantially reduced pathology in the rCIA model.
RVX-297 Inhibits Progression of Pathology in the Mouse Collagen-Induced Arthritis Model. The ability of RVX-297 to counter autoimmune disease progression was further characterized and confirmed in the mouse CIA (mCIA) model (Inglis et al., 2007). Collagen injections increased the CAS as expected (Supplemental Fig. 1A). Notably, treatment with RVX-297 at both 75 and $150 \mathrm{mg} / \mathrm{kg}$ and the positive control dexamethasone all prevented significant increases in CAS compared with the arthritic vehicle-treated cohort from day 2 forward (Supplemental Fig. 1A) (ANOVA with Tukey's test). Effects of RVX-297 were dose dependent. Effects of dexamethasone and RVX-297 $150 \mathrm{mg} / \mathrm{kg}$ were so profound that the CAS was not distinguishable from that of naive animals from day 3 on (ANOVA with Tukey's test). Similar to rCIA, histopathology scores in all paws, knee, and six-joint mean were significantly reduced with RVX-297 or dexamethasone treatment compared with vehicle-treated arthritic controls (Table 4; Student's $t$ test). In addition, significantly lower anti-collagen II IgG serum levels were measured in mice treated with RVX-297 $150 \mathrm{mg} / \mathrm{kg}$ compared with vehicle-treated controls (Supplemental Fig. 1B) (ANOVA with

TABLE 2

RVX-297 suppresses histopathological parameters in rCIA

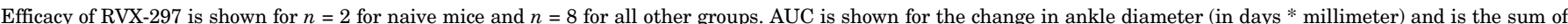

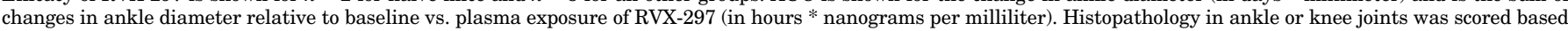

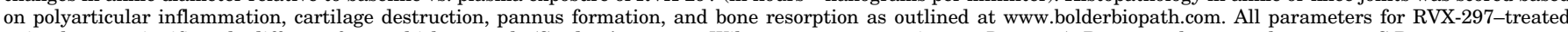
animals were significantly different from vehicle controls (Student's $t$ test or Wilcoxon nonparametric test, $P<0.05$ ). Data are shown as the mean \pm S.D.

\begin{tabular}{|c|c|c|c|c|c|c|}
\hline Group & Dose (mg/kg BID) & $\begin{array}{l}\text { Mean AUC Change } \\
\text { in Ankle Diameter }\end{array}$ & $\begin{array}{c}\text { Inhibition in AUC } \\
\text { Change in Ankle Diameter }(\%)\end{array}$ & $\begin{array}{c}\text { Reduction in Ankle } \\
\text { Histopathology Score (\%) }\end{array}$ & $\begin{array}{c}\text { Reduction in Knee } \\
\text { Histopathology Score }(\%)\end{array}$ & $\begin{array}{l}\text { RVX-297 Plasma Exposure } \\
\text { AUC }(0-12)(\mathrm{hr} * \mathrm{ng} / \mathrm{ml})\end{array}$ \\
\hline Naive & NA & 0.05 & NA & NA & $\mathrm{NA}$ & $\mathrm{NA}$ \\
\hline Vehicle & 0 & $7.5 \pm 1.4$ & NA & $0 \pm 4.5$ & $0 \pm 33$ & NA \\
\hline RVX-297 & 25 & $2.6 \pm 2.3$ & 66 & $26 \pm 25$ & $83 \pm 30$ & 23,165 \\
\hline RVX-297 & 50 & $0.9 \pm 1.8$ & 89 & $64 \pm 29$ & $86 \pm 17$ & 52,145 \\
\hline RVX-297 & 75 & $0.7 \pm 1.5$ & 92 & $64 \pm 20$ & $94 \pm 10$ & 86,452 \\
\hline
\end{tabular}

NA, not applicable. 
TABLE 3

RVX-297 decreases cytokine gene expression and protein levels in the ankle of the rCIA model

Percent reduction in gene expression $(n=7)$ or protein levels $(n=4)$ in the ankle of rCIA animals treated with $75 \mathrm{mg} / \mathrm{kg}$ BID RVX-297 vs. vehicle-treated controls. Data are shown as the mean \pm S.D. Statistical significance compared with the vehicletreated controls was determined using a Student's $t$ test.

\begin{tabular}{lclc}
\hline \multicolumn{1}{c}{ Gene } & $\begin{array}{c}\text { Reduction in Gene } \\
\text { Expression in Ankles (\%) }\end{array}$ & Protein & $\begin{array}{c}\text { Reduction in Ankle } \\
\text { Joint Protein (\%) }\end{array}$ \\
\hline IL-1 $\beta$ & $77 \pm 16^{* * *}$ & IL-6 & $52 \pm 28^{*}$ \\
MMP3 & $60 \pm 21^{* * *}$ & VCAM-1 & $33 \pm 4^{* * *}$ \\
MMP13 & $37 \pm 16^{* * *}$ & & \\
RANKL & $70 \pm 6^{* * *}$ & & \\
\hline
\end{tabular}

$* P<0.05 ; * * * P<0.001$.

Tukey's test). In fact, anti-collagen II IgG levels were statistically indistinguishable between the naive cohort and the cohort of mice treated with RVX-297 $150 \mathrm{mg} / \mathrm{kg}$. Lower levels of anti-collagen II IgG indicate that RVX-297 inhibited B-cell activation and antibody production to moderate the response to collagen injection. Thus, RVX-297 ameliorates CASs, histopathology scores, and serum IgG levels in the mCIA model.

RVX-297 Inhibits Pathology in the mCAIA Model. The mCAIA model offers a distinct advantage over CIA as arthritic pathology develops in the absence of adjuvants (i.e., complete or incomplete Freund's adjuvant). Collagen antibody injections caused a significant increase in CAS that developed with time in vehicle-treated arthritic mice compared with naive mice (Fig. 5) (ANOVA with Tukey's test, $P<0.001$ from day 4 on), an observation analogous to that in the CIA models. Treatment was initiated after disease symptoms were established. RVX-297 prevented the progression of clinical manifestations in this model by day 5 , as indicated through the CAS (Fig. 5) (ANOVA with Tukey's test vs. vehicle). Dexamethasone, the positive control, restored CAS in CAIA mice to the level of naive animals (Fig. 5) (ANOVA with Tukey's test). Histopathology scores were also significantly reduced by RVX297 compared with vehicle-treated controls in both CIA and CAIA mice (Table 4; Student's $t$ test). Thus, RVX-297 and dexamethasone both produced clear and significant beneficial effects on the clinical parameters and histology associated with collagen antibody-induced arthritis in mice. In all, the three autoimmune arthritis models presented here show that BET proteins play a principal role in the development and maintenance of pathology mimicking arthritis by contributing to the processes of inflammation, cartilage destruction, bone resorption, and production of antibodies underlying the autoimmune process. RVX-297, a BETi, can ameliorate arthritis symptoms through the downregulation of factors derived from multiple contributing cell types that propagate or promote the disorder.

RVX-297 Diminishes EAE Pathology. Evaluation of RVX-297 treatment was extended to the EAE mouse model, a $\mathrm{T}$ cell-mediated autoimmune model that shares many clinical and histopathological features with human MS. FTY720 (fingolimod), an approved drug for MS that is efficacious in the mouse EAE model (Kataoka et al., 2005), was used as a comparator treatment with a mechanism of action distinct from BETi. RVX297 or FTY720 treatment was initiated concurrent with the induction of disease in a prevention model (Fig. 6A) or after the establishment of EAE in a therapeutic regimen (Fig. 6B). The impact of treatment on EAE clinical scores (Fig. 6C and D), the percent change in body weight (Fig. $6 \mathrm{E}$ and F), and EAEdependent changes in spinal cord histology (Fig. 6G and H; Table 5) were all assessed.

Within the prevention model, the EAE clinical score began to increase significantly from baseline around day 15 post immunization (Figure 6C), which was suppressed remarkably by RVX-297 or FTY720 (ANOVA with Tukey's test, $P<0.05$ from day 15 on) (Fig. 6C). In the therapeutic regimen, RVX297 countered the increase in EAE clinical scores in a dosedependent manner (Fig. 6D) (ANOVA with Tukey's test: $P<0.05$ by day 2 on for RVX-297 $125 \mathrm{mg} / \mathrm{kg}$; by day 9,12 , 13, and 14 for RVX-297 $75 \mathrm{mg} / \mathrm{kg}$; and by day 4 on for FTY720). RVX-297 had a similar impact on clinical scores as FTY720, an MS therapeutic agent, in both the prevention and the therapeutic regimens.

Weight loss is a widely recognized symptom of EAE onset (Emerson et al., 2009). Here, the body weight of the vehicletreated cohort began to deviate significantly from that of naive animals by day 17 after immunization in the prevention study (Fig. 6E) (ANOVA with Tukey's test) and by day 13 in the therapeutic study (Fig. 6F) (ANOVA with Tukey's test). Treatment with both RVX-297 $75 \mathrm{mg} / \mathrm{kg}$ and FTY720 prevented substantial losses in body weight induced by EAE (Fig. 6E) (ANOVA with Tukey's test). Treatment with RVX-297 at $125 \mathrm{mg} / \mathrm{kg}$ accelerated weight loss, because it was not well tolerated. Dose was adjusted to $100 \mathrm{mg} / \mathrm{kg}$ BID on day 18, and weight recovery was apparent by day 21 . Statistically, the weights of the RVX-297 $100 \mathrm{mg} / \mathrm{kg}$ cohort

TABLE 4

RVX-297 reduces histopathology in mouse models of arthritis

Histopathology was assessed and scored based on polyarticular inflammation, cartilage destruction, pannus formation, and bone resorption (outlined at www.bolderbiopath.com). Doses were delivered twice per day in 10 mice/group. Results are presented as percent reduction compared with vehicle-treated mCIA or mCAIA controls, and data are shown as the mean \pm S.D. Statistical significance compared with the vehicle-treated controls was determined using a Student's $t$ test.

Percent reduction in histopathology score

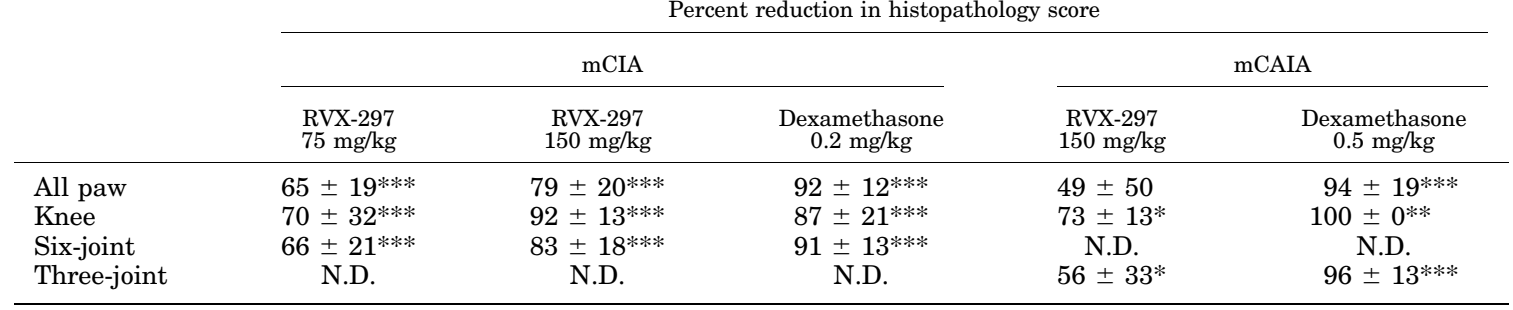

N.D., not determined.

$* P<0.05 ; * * P<0.01 ; * * * P<0.001$. 


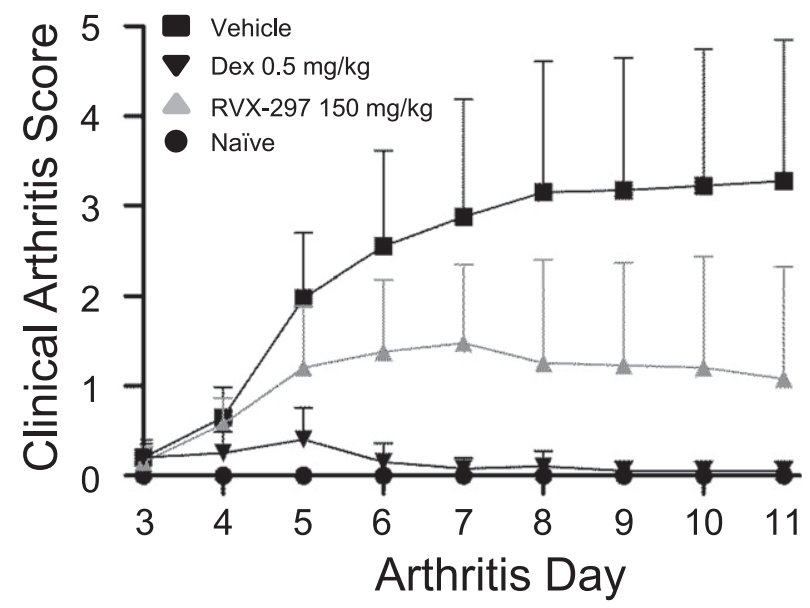

Fig. 5. RVX-297 improves CAS in the mCAIA model. Oral RVX-297 dosing twice a day was initiated after the establishment of CAIA in female BALB/cAnNHsd mice. CAS (scored 0-5; www.bolderbiopath.com) are presented as the mean \pm S.D. $[n=10$ mice/group (except for naive mice, $n=5)$ ]. CAS rapidly increased after CAIA establishment (ANOVA with Tukey's test, $P<0.001$, arthritis plus vehicle vs. naive mice from day 4 on). RVX-297 treatment significantly reduced the CAS in CAIA mice vs. vehicle-treated mice by day 5 on (ANOVA with Tukey's test, $P<0.05$ ). Dexamethasone (dex) treatment suppressed increases in the CAS in CAIA mice (ANOVA with Tukey's test $P<0.05$ day 4 on vs. vehicle-treated mice; no significant difference vs. naive from day 4 on).

were indistinguishable from the naive, RVX-297 $75 \mathrm{mg} / \mathrm{kg}$, and FTY720 cohorts on days 20-24 (Fig. 6E) (ANOVA with Tukey's test). Our results demonstrate that treatment with RVX-297 and FTY720 had clear and sustained effects on outward manifestations of EAE in both preventative and therapeutic regimens.

Histopathological examination of spinal cords revealed that vehicle-treated mice had prominent hallmarks of EAE pathology (Fig. 6G and H, left panels). Inflammatory foci were large, coalescing, and present around blood vessels in the leptomeninges as well as deep in the white matter. Demyelination was prevalent in all spinal cord sections, and large numbers of apoptotic cells were apparent. Strikingly, mice treated with RVX-297 in the prophylactic protocol had no histologic signs of EAE in the spinal cord, no inflammatory foci, no demyelination, and no apoptotic cells in any of the histologic sections, and were indistinguishable from naive mice (Fig. 6G; Table 5). The impact of RVX-297 on clinical scores (Fig. 6C) is mirrored in the marked histologic improvements observed with the preventative RVX-297 treatment of EAE mice. In the therapeutic regimen, microscopic evaluation showed that mice treated with RVX-297 and FTY720 had significantly lower inflammation, as evidenced by a reduction in the number of inflammatory foci, an improvement in demyelination scores, and a sparsity of apoptotic cells in the spinal cord compared with vehicletreated animals (Fig. 6H; Table 5). Collectively, RVX-297 was effective in reducing disease severity and improving clinical parameters in mice when dosed after disease onset and was highly efficacious in reducing EAE development when dosed concurrently with immunization.

Proinflammatory cytokines are important contributors to the establishment and progression of autoimmune diseases. The gene expression of critical inflammatory cytokines in the spinal cord was examined. mRNA levels of IL-6, IL-17, MCP-1,
GM-CSF, and TNF $\alpha$ mRNAs were all significantly lower in RVX-297-treated EAE mice compared with vehicle-treated controls (Fig. 7) (Student's $t$ test), suggesting that treatment prevented localized inflammation in the spinal cord. RVX-297 treatment also increased Hist2h2be gene expression in the spinal cord, a known BETi target engagement marker (Delmore et al., 2011; Mertz et al., 2011), more than 4-fold versus vehicle-treated controls (data not shown). Myelin basic protein-specific $\mathrm{CD}^{+} \mathrm{T}$ cells mediate $\mathrm{EAE}$ pathology by migrating from the periphery into the CNS (Furtado et al., 2008; O'Connor et al., 2008). Here, the percentage of CD4 ${ }^{+}$ cells producing specific proinflammatory cytokines was quantified in the brain and spinal cord to determine the extent of infiltration into the CNS. RVX-297 significantly lowered the abundance of IL-6, GM-CSF, and IFN $\gamma \mathrm{CD}^{+}$cells, indicating a reduction in CNS infiltration (Fig. 8). To examine cytokine production, lymphocytes were isolated from EAE mice that had been treated for 11 days with either vehicle or RVX-297, which were rechallenged with MOG peptides 35-55 ex vivo. Inflammatory cytokine secretion into the culture media (IL-17a, IL-6, TNF $\alpha$, and IFN $\gamma$ ) was substantially reduced compared with lymphocytes isolated from vehicle-treated controls (Table 6). Together, observations in EAE mice demonstrate that BETi modulates key mediators of inflammation that drive the progression of autoimmunity from multiple contributing cell types and can alleviate EAE pathology even after the onset of disease.

\section{Discussion}

Small-molecule pan-BETis have been useful in delineating roles for BET BDs in inflammatory processes and autoimmune disorders (Nicodeme et al., 2010; Barrett et al., 2014; Zhang et al., 2015; Chen et al., 2016). Whereas most BETis in development have similar affinity for both (i.e., pan) BET protein BDs, RVX-297 is a novel, orally bioavailable BETi with selectivity for BD2 (Kharenko et al., 2016). Apabetalone is another BD2-selective BETi currently in clinical trials for cardiovascular disease (McLure et al., 2013); the antiinflammatory properties of apabetalone have been demonstrated in a mouse model of atherosclerosis (Jahagirdar et al., 2014). To extend the understanding of the anti-inflammatory properties of RVX-297, we examined the activity of RVX-297 in vitro and in five preclinical in vivo models of autoimmune pathology including, the mouse LPS, rCIA, mCIA, mCAIA, and mouse EAE models. RVX-297 reduced the response to inflammatory stimuli in macrophages, B cells, $\mathrm{T}$ cells, and fibroblasts, which is consistent with the displacement of BET proteins from chromatin. In vivo, RVX-297 drastically reduced disease symptoms in rodent models of endotoxemia and autoimmune disease. In addition, RVX-297 regulated inflammatory gene expression in disease-contributing cell types.

Responses to inflammatory stimuli are complex. The activation of inflammatory gene expression involves epigenetic restructuring of chromatin and the associated regulatory elements to alter the transcriptome (Brown et al., 2014). Current evidence suggests that anti-inflammatory properties of BETi are mediated through the displacement of BET proteins from acetylation marks on chromatin, disrupting the assembly of transcriptional machinery and the subsequent expression of BET sensitive genes (Nicodeme et al., 2010). BETis also effectively suppress inflammatory responses 
A

Schematic Representation of Mouse EAE Study Design: Prevention

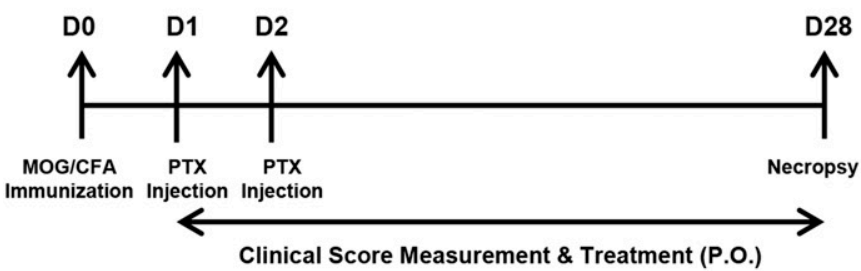

C

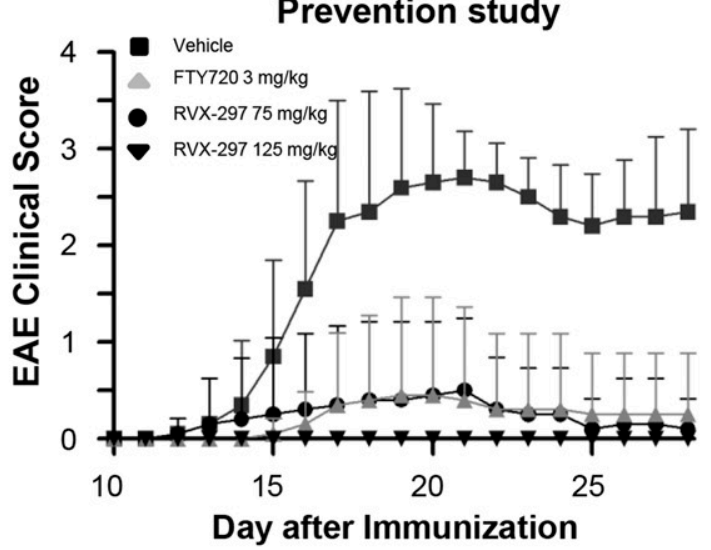

$\mathbf{E}$

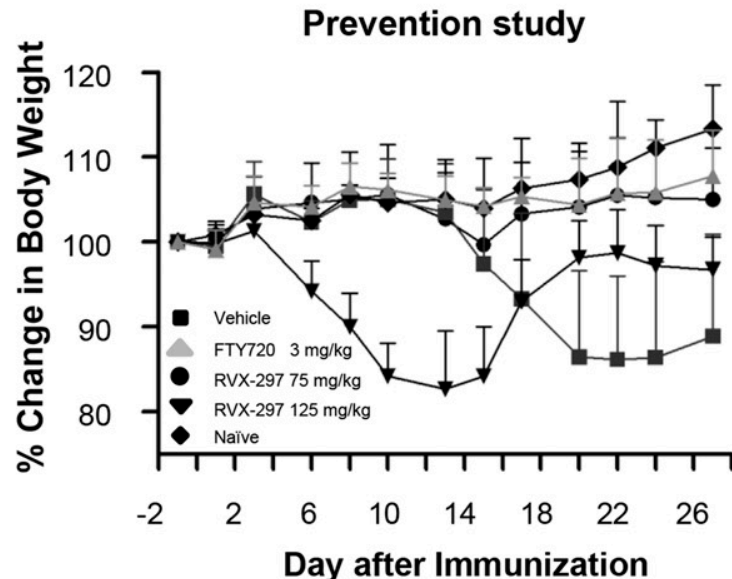

G

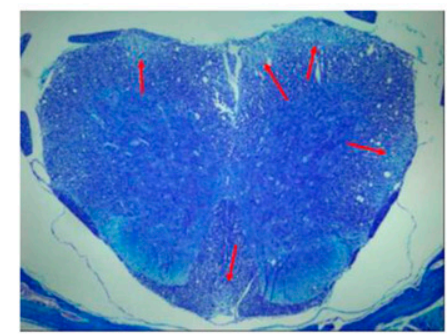

Vehicle

\section{Prevention study}

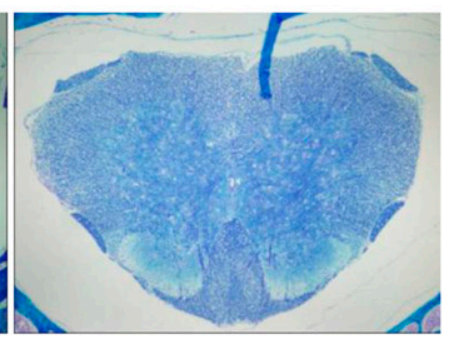

RVX-297, $75 \mathrm{mg} / \mathrm{kg}$

B Schematic Representation of Mouse EAE Study Design: Therapeutic

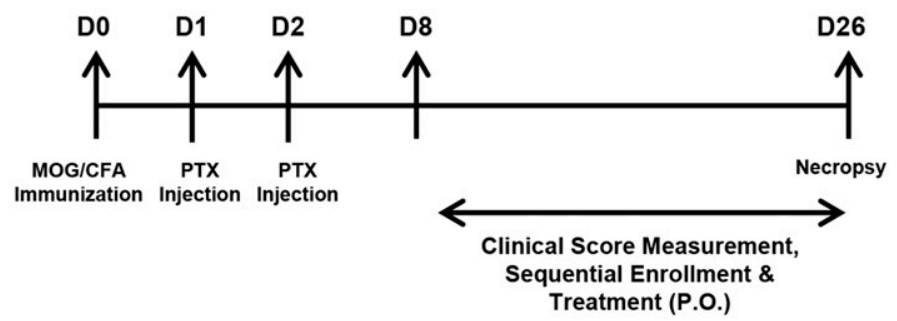

D Therapeutic study

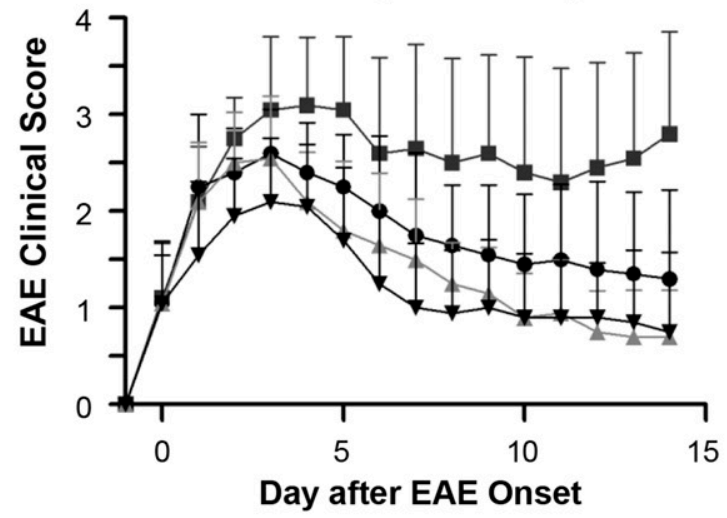

$\mathbf{F}$

Therapeutic study

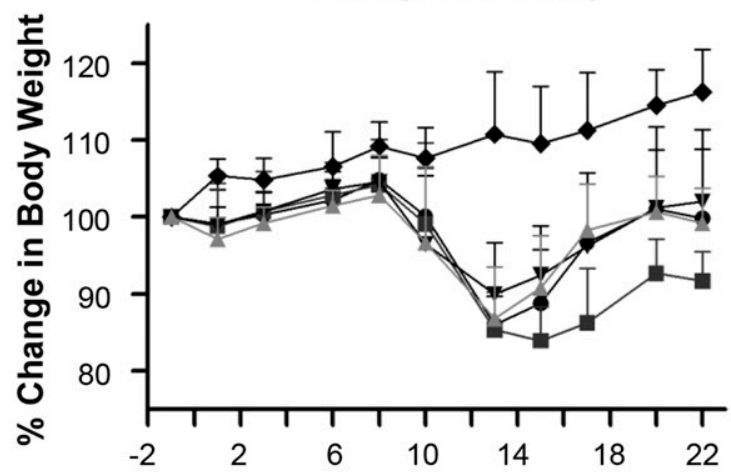

Day after Immunization

H

Therapeutic study

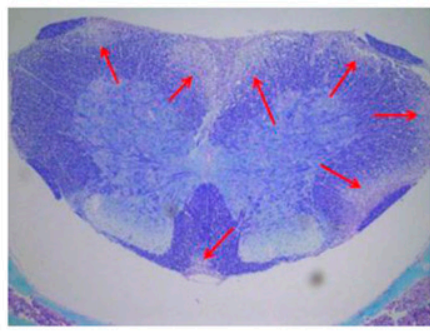

Vehicle

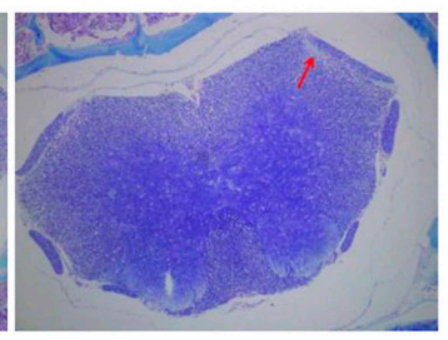

RVX-297, $125 \mathrm{mg} / \mathrm{kg}$

Fig. 6. RVX-297 ameliorates clinical manifestations and histopathology associated with mouse EAE in both prevention and therapeutic regimens. (A and B) Prevention and therapeutic study designs, respectively. In both studies, mice were evaluated prior to initiation (day -1), and EAE was induced on day 0. (C) RVX-297 and FTY720 both prevented the rise in EAE clinical score observed 14 days after EAE induction; data are the mean \pm S.D. At day 15, the clinical scores for the RVX-297 $125 \mathrm{mg} / \mathrm{kg}$ and FTY720 treatment groups differed significantly from those of the vehicle-treated cohort (ANOVA with Tukey's test, $P<0.05$ ), and by day 16 for the RVX-297 $75 \mathrm{mg} / \mathrm{kg}$ cohort. (D) RVX-297 and FTY720 had a therapeutic effect on EAE clinical scores. Data are presented as the mean \pm S.D. Beginning on day 2 , administration of RVX-297 $125 \mathrm{mg} / \mathrm{kg}$ significantly decreased the EAE clinical score compared with vehicle (ANOVA with Tukey's test, $P<0.05$ ). From day 4 on, FTY720 effectively decreased the EAE clinical score compared with vehicle. RVX-297 $75 \mathrm{mg} / \mathrm{kg}$ differed significantly from vehicle on days 9, 12-14 (ANOVA with Tukey's test, $P<0.05$ ). (E) RVX-297 $75 \mathrm{mg} / \mathrm{kg}$ and FTY720 prevented EAEassociated weight loss. Comparison of the percentage change in body weight observed for the RVX-297 75 mg/kg, FTY720, and naive cohorts revealed no 
TABLE 5

RVX-297 and FTY720 reduce inflammation, demyelination, and apoptosis in the spinal cords of EAE mice

Histology was assessed in the cervical, thoracic, and lumbar regions of the spinal cords of EAE mice. Each parameter is presented as an average from these regions compared with vehicle-treated disease controls. Student's $t$ test ( $P$ values included in table) was used to determine statistical significance compared with vehicle-treated EAE mice $[n=10$ mice/group (except for naive mice, $n=3)]$. Data are the mean \pm S.D.

\begin{tabular}{|c|c|c|c|c|c|c|}
\hline Group Treatment & Inflammatory Foci & $P$ value & Demyelination (LFB) & $P$ value & Apoptotic Cells & $P$ value \\
\hline \multicolumn{7}{|c|}{ Preventative treatment regimen } \\
\hline Naive & $0.0 \pm 0.0$ & $<0.0001$ & $0.0 \pm 0.0$ & $<0.0001$ & $0.0 \pm 0.0$ & $<0.0001$ \\
\hline Vehicle & $4.3 \pm 3.2$ & NA & $1.6 \pm 1.0$ & NA & $2.7 \pm 2.5$ & NA \\
\hline FTY720 (3 mg/kg) & $0.3 \pm 1.0$ & $<0.0001$ & $0.0 \pm 0.2$ & $<0.0001$ & $0.1 \pm 0.4$ & $<0.0001$ \\
\hline RVX-297 (75 mg/kg) & $1.5 \pm 2.9$ & 0.0004 & $0.1 \pm 0.3$ & $<0.0001$ & $0.5 \pm 0.9$ & $<0.0001$ \\
\hline RVX-297 (125 mg/kg) & $0.1 \pm 0.6$ & $<0.0001$ & $0.0 \pm 0.0$ & $<0.0001$ & $0.0 \pm 0.0$ & $<0.0001$ \\
\hline \multicolumn{7}{|c|}{ Therapeutic treatment regimen } \\
\hline Naive & $0.0 \pm 0.0$ & $<0.0001$ & $0.0 \pm 0.0$ & $<0.0001$ & $0.0 \pm 0.0$ & $<0.0001$ \\
\hline Vehicle & $3.9 \pm 2.4$ & NA & $1.6 \pm 0.8$ & NA & $2.0 \pm 1.8$ & NA \\
\hline FTY720 (3 mg/kg) & $0.8 \pm 0.9$ & $<0.0001$ & $0.8 \pm 0.5$ & $<0.0001$ & $0.8 \pm 0.9$ & 0.002 \\
\hline RVX-297 (75 mg/kg) & $1.7 \pm 1.2$ & $<0.0001$ & $1.1 \pm 0.8$ & 0.02 & $0.5 \pm 1.0$ & 0.0002 \\
\hline RVX-297 (125 mg/kg) & $1.6 \pm 1.9$ & 0.0001 & $0.9 \pm 0.8$ & 0.002 & $0.3 \pm 0.6$ & $<0.0001$ \\
\hline
\end{tabular}

LFB, Luxol fast blue; NA, not applicable.

mediated by nuclear factor- $\kappa$-light-chain-enhancer of activated B cells (NF- $\kappa$ B) (Brown et al., 2014), a proinflammatory transcription factor and master regulator of inflammatory and immune responses (for review, see Baltimore, 2011; Smale, 2011). For example, the NF- $\kappa$ B pathway is activated by LPS, and the pan-BETi I-BET762 has been shown to prevent or diminish the incidence of death in mice given lethal doses of LPS (Nicodeme et al., 2010). These data indicate that the modulation of BET protein activity can attenuate the cytokine storm associated with endotoxemic shock or sepsis. Genetic and chemical approaches have demonstrated the critical role of BRD2 and BRD4 in the regulation of inflammatory cytokine expression in macrophages (Belkina et al., 2013). In our study, RVX-297 blunts LPS-induced endotoxemia in mice, resulting in reduced numbers of serum cytokines, inflammatory markers (Fig. 3A; Supplemental Table 1), and LPS-induced splenic gene expression of IL-6 and IL-17 (Fig. 3B). Our results are consistent with studies linking BETi with the suppression of exaggerated inflammatory responses tied to cytokine production and NF- $\kappa \mathrm{B}$ activation (Huang et al., 2009; Belkina et al., 2013).

Because RVX-297 mediated the suppression of key factors that drive autoimmune disease in LPS-treated mice, studies were extended to preclinical autoimmune models. Rodent models of RA provide insights into the perpetuation and treatment of pathogenic joint inflammation. The CIA and CAIA models are characterized by hallmarks of arthritis, including measurable polyarticular inflammation, marked cartilage destruction in association with pannus formation, and mild to moderate bone resorption and periosteal bone proliferation (Bendele, 2001; Williams, 2004). In three arthritis models, disease indices were dramatically reduced in RVX-297-treated animals, resulting in the maintenance of joint structure and integrity, likely arising from decreased production of cytokines and inflammatory mediators. The pan-BETi JQ1 is also efficacious in the mCIA model, stressing a role for BET proteins in the inflammatory response, the production of autoantibodies, and joint damage arising in this model (Zhang et al., 2015). In addition, a fundamental role for IL-17 in the CAIA model disease development has been demonstrated (Plum et al., 2009). The ability of RVX-297 to downregulate IL-17 production (Tables 1 and 6; Supplemental Table 1) may contribute to the efficacy of this compound.

$\mathrm{T}_{\mathrm{H}} 17$ cells are fundamental in promoting human autoimmune disease, which is recapitulated in mouse models (Nakae et al., 2003; Komiyama et al., 2006). $\mathrm{T}$ cells of both $\mathrm{T}_{\mathrm{H}} 1$ and $\mathrm{T}_{\mathrm{H}} 17$ lineages are involved in generating disease conditions in EAE (Kroenke et al., 2008). Cytokines IL-23, IL-6, and IL-17, which are critical for $\mathrm{T}_{\mathrm{H}} 1$ and $\mathrm{T}_{\mathrm{H}} 17$ differentiation and the production of these $\mathrm{T}$ cells, play a fundamental and nonredundant role in EAE progression (Thakker et al., 2007). Therefore, pharmacological suppression of these cytokines, or $\mathrm{T}_{\mathrm{H}} 17$ differentiation, has therapeutic potential in $\mathrm{T}$ cellmediated autoimmune disorders. Further, the neutralization of IL-17 through targeting of the protein or its receptor prevents the development of EAE (Hofstetter et al., 2005). Previous studies have demonstrated that JQ1 ameliorates EAE symptoms and established that BET BDs are critical in the differentiation and function of $\mathrm{T}_{\mathrm{H}} 17$ cells (Mele et al., 2013). RVX-297 suppressed EAE pathology when administered to mice either prophylactically or therapeutically. In addition, the production of multiple cytokines in vivo (Fig. 7) and ex vivo (Table 6) was suppressed by RVX-297. In vivo efficacy was comparable or superior to that of the S1P1 agonist FTY720 (fingolimod), an approved therapeutic agent for the treatment of MS. RVX-297 inhibits $\mathrm{T}_{\mathrm{H}} 1$ and $\mathrm{T}_{\mathrm{H}} 17$ responses, both of which play pivotal roles in the development and progression of MS and other autoimmune indications. Additional analysis of cytokine production indicates that RVX-297 affects multiple contributors to autoimmune pathology. Importantly, RVX-297 decreased $\mathrm{CD} 4^{+}$cells producing proinflammatory cytokines in the brains and spinal cords of EAE mice (Fig. 8), indicating reduced infiltration of $\mathrm{T}$ cells into the

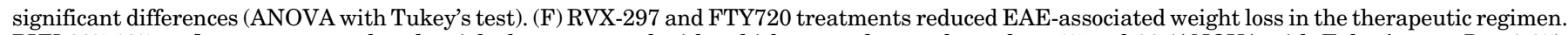

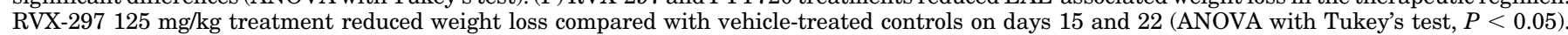

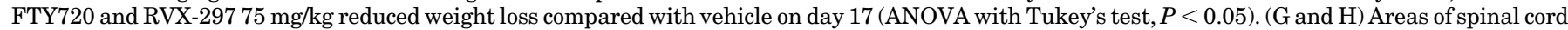

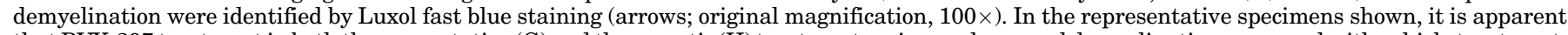

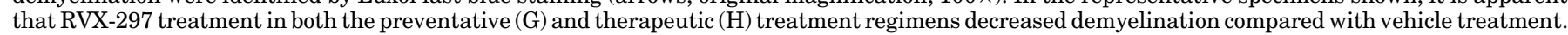
MOG, myelin oligodendrocyte glycoprotein; PTX, pertussis toxin; CFA, complete Freund's adjuvant. 


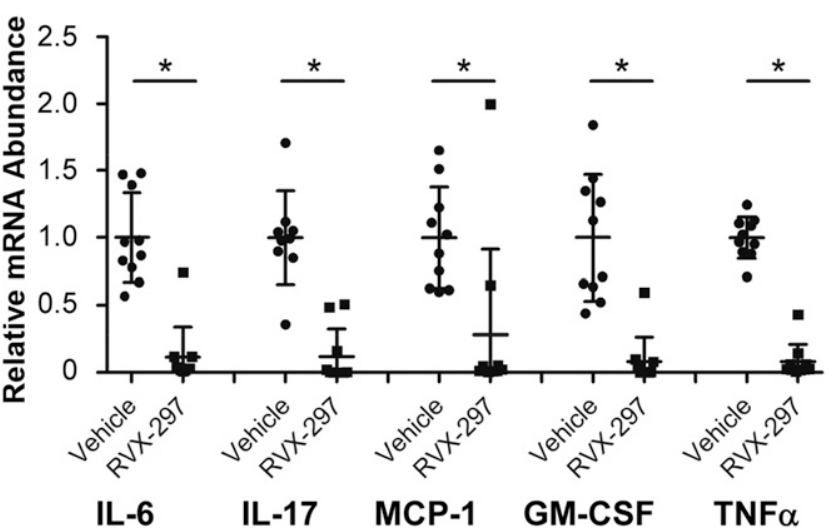

Fig. 7. RVX-297 decreases inflammatory gene expression in the spinal cords of EAE mice. Inflammatory gene expression (IL-6, IL-17, MCP-1, GM-CSF, and $\mathrm{TNF} \alpha$ ) in spinal cords of EAE mice treated orally twice a day with vehicle or $100 \mathrm{mg} / \mathrm{kg}$ RVX-297 in the preventative regimen was determined by real-time PCR. Data are the mean of either 9 or 10 animals/group. Error bars represent the S.D. Statistical significance was determined with a Student's $t$ test $(* P<0.05)$.

CNS. These results underscore the effects of BETi in the periphery to alleviate the effects in the CNS (Tzartos et al., 2008). Although RVX-297 was effective in reducing IL-17producing $\mathrm{T}$ cells in the CNS, more profound effects were reported with JQ1 (Mele et al., 2013). This variance may be due to differences in compound affinities for BET BDs. Results from this study and from other laboratories have shown that BET BD inhibition protects mice from autoimmunity (Belkina et al., 2013; Mele et al., 2013; Zhang et al., 2015). Therefore, BETi holds promise for the treatment of autoimmunity, even after disease symptoms have been established.

A variety of pharmacological therapies has been approved for the treatment of autoimmune disorders. Antibodies that capture or neutralize key cytokines to promote

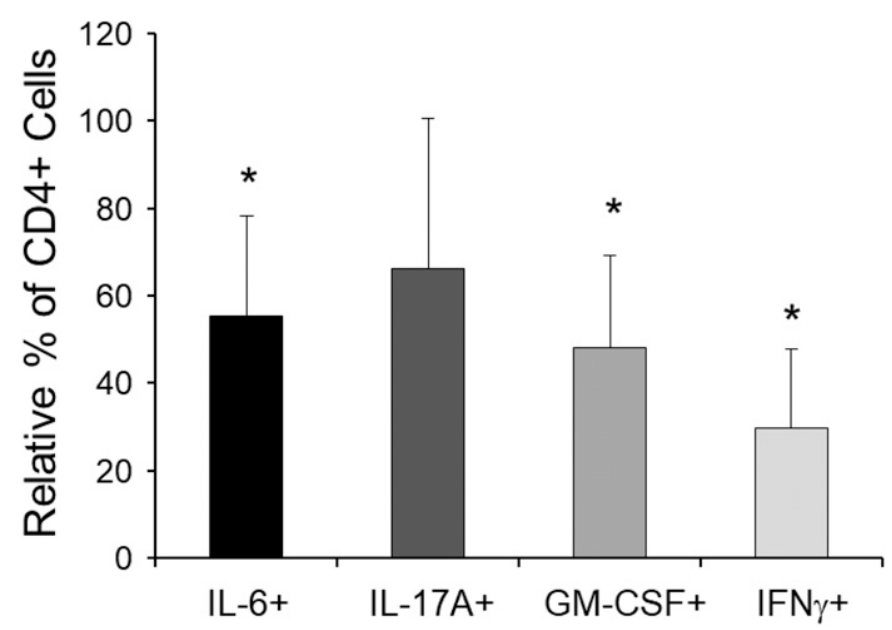

Fig. 8. RVX-297 prevents $\mathrm{CD} 4^{+}$cell infiltration and cytokine production in the CNS of EAE mice. Activated $\mathrm{CD} 4^{+} \mathrm{T}$ cells producing the indicated proinflammatory cytokines in the brain and spinal cord of EAE mice treated with vehicle or $125 \mathrm{mg} / \mathrm{kg}$ RVX-297 were isolated. The $\mathrm{CD} 4^{+} \mathrm{T}$ cells were further stimulated with PMA, ionomycin, and brefeldin for 4-5 hours and then quantified by flow cytometry. Data are the mean percent reductions in cells from mice treated with RVX-297 vs. mice treated with vehicle; $n=6$ mice/group. Error bars represent the S.D. Statistical significance vs. vehicle-treated controls was determined with a Student's $t$ test $(* P<0.05)$.
TABLE 6

RVX-297 decreases ex vivo cytokine production by lymphocytes isolated from EAE mice

Lymphocytes were isolated from EAE mice treated with RVX-297 ( $n=3$ ) or vehicle $(n=3)$ for 11 days. Independent cultures established from each mouse were rechallenged with MOG peptides 35-55 for 72 hours and secreted cytokine levels determined through cytometric bead assays run in triplicate. Data represent the mean percent reduction in cytokines produced by cells from RVX-297-treated mice compared with cells from vehicle-treated controls, with variability presented as the S.D. Statistical significance was determined with a Student's $t$ test.

\begin{tabular}{lllc}
\hline \multirow{2}{*}{ Protein } & \multicolumn{3}{c}{ MOG $(\mu \mathrm{g} / \mathrm{ml})$} \\
\cline { 2 - 4 } & \multicolumn{1}{c}{0.7} & \multicolumn{2}{c}{2.2} \\
\hline & $\%$ reduction \\
IL-17a & $67 \pm 9^{* *}$ & $80 \pm 7^{* *}$ & $73 \pm 11^{* * *}$ \\
IL-6 & $37 \pm 13^{*}$ & $58 \pm 12^{* *}$ & $64 \pm 4^{* *}$ \\
TNF $\alpha$ & $50 \pm 15^{*}$ & $79 \pm 4^{* *}$ & $79 \pm 5^{* * *}$ \\
IFN $\gamma$ & $93 \pm 1$ N.S. & $99 \pm 0^{*}$ & $99 \pm 0^{* * *}$ \\
\hline
\end{tabular}

N.S., not significant; $* P<0.05 ; * * P<0.01 ; * * * P<0.001$.

immunosuppression are prevalent (for review, see Chan and Carter, 2010). Because autoimmunity causing host tissue damage and antimicrobial immunity involves the same processes, infection is a concern due to the immunosuppressive nature of these drugs (for review, see Caspi, 2008). Therefore immunosuppressive therapies are often not well suited for long-term treatment. Of note, the BD2-selective compound apabetalone had no effect on the incidence of infestations or infections in a 6-month clinical trial of patients with cardiovascular disease (Nikolic et al., 2015; Nicholls et al., 2016). This outcome may be a result of suppressing inflammatory processes to levels that do not entirely impair immune function, thereby avoiding the risk of infection.

In contrast to targeting single entities in inflammatory or autoimmune pathways, BETi simultaneously downregulates multiple inflammatory processes. Concurrent targeting of contributors to inflammation from a variety of immune cell types sets BETi apart from current therapies that act on a single inflammatory component or process. Studies indicate that BET protein BDs function cooperatively (Schroder et al., 2012). How BD1 is impacted, directly or indirectly, by BD2-selective inhibition requires further investigation. Our results suggest that BD2-selective BET inhibitors are sufficient to ameliorate autoimmune pathology and may dampen detrimental immune responses without adversely compromising immune function. Future studies will be required to determine whether RVX-297 BETi is clinically effective in autoimmune conditions.

\section{Acknowledgments}

The authors thank S. Wasiak, S. Stotz, and B. Rakai for stimulating discussions and critical review of the manuscript. The authors are also grateful to E. Daze for assistance with graphing.

\section{Authorship Contributions}

Participated in research design: Jahagirdar, Attwell, Marusic, Bendele, Shenoy, McLure, Hansen, Wagner, Young, Wong, and Kulikowski.

Conducted experiments: Attwell, Marusic, Bendele, Shenoy, Norek, $\mathrm{Yu}$, and Tobin.

Performed data analysis: Jahagirdar, Attwell, Marusic, Bendele, Shenoy, McLure, Norek, Yu, and Tobin.

Wrote or contributed to the writing of the manuscript: Jahagirdar, Attwell, and Gilham. 


\section{References}

Baltimore D (2011) NF-кB is 25. Nat Immunol 12:683-685.

Bandukwala HS, Gagnon J, Togher S, Greenbaum JA, Lamperti ED, Parr NJ, Molesworth AM, Smithers N, Lee K, Witherington J, et al. (2012) Selective in hibition of CD4 + T-cell cytokine production and autoimmunity by BET protein and c-Myc inhibitors. Proc Natl Acad Sci USA 109:14532-14537.

Bandukwala HS, Wu Y, Feuerer M, Chen Y, Barboza B, Ghosh S, Stroud JC, Benoist C, Mathis D, Rao A, et al. (2011) Structure of a domain-swapped FOXP3 dimer on DNA and its function in regulatory T cells. Immunity 34:479-491.

Barrett E, Brothers S, Wahlestedt C, and Beurel E (2014) I-BET151 selectively regulates IL-6 production. Biochim Biophys Acta 1842:1549-1555.

Baumgart DC and Carding SR (2007) Inflammatory bowel disease: cause and immunobiology. Lancet 369:1627-1640.

Belkina AC, Nikolajczyk BS, and Denis GV (2013) BET protein function is required for inflammation: Brd2 genetic disruption and BET inhibitor JQ1 impair mouse macrophage inflammatory responses. J Immunol 190:3670-3678.

Bendele A (2001) Animal models of rheumatoid arthritis. J Musculoskelet Neuronal Interact 1:377-385.

Biswas P, Delfanti F, Bernasconi S, Mengozzi M, Cota M, Polentarutti N, Mantovani A, Lazzarin A, Sozzani S, and Poli G (1998) Interleukin-6 induces monocyte chemotactic protein-1 in peripheral blood mononuclear cells and in the U937 cell line. Blood 91:258-265.

Bittner S, Afzali AM, Wiendl H, and Meuth SG (2014) Myelin oligodendrocyte glycoprotein (MOG35-55) induced experimental autoimmune encephalomyelitis (EAE) in C57BL/6 mice. J Vis Exp (86):e51275.

Brown JD, Lin CY, Duan Q, Griffin G, Federation A, Paranal RM, Bair S, Newton G, Lichtman A, Kung A, et al. (2014) NF-кB directs dynamic super enhancer formation in inflammation and atherogenesis. Mol Cell 56:219-231.

Carr MW, Roth SJ, Luther E, Rose SS, and Springer TA (1994) Monocyte chemoattractant protein 1 acts as a T-lymphocyte chemoattractant. Proc Natl Acad Sci USA 91:3652-3656.

Caspi RR (2008) Immunotherapy of autoimmunity and cancer: the penalty for success. Nat Rev Immunol 8:970-976.

Chan AC and Carter PJ (2010) Therapeutic antibodies for autoimmunity and inflammation. Nat Rev Immunol 10:301-316.

Chen K, Campfield BT, Wenzel SE, McAleer JP, Kreindler JL, Kurland G, Gopal R, Wang T, Chen W, Eddens T, et al. (2016) Antiinflammatory effects of bromodomain and extraterminal domain inhibition in cystic fibrosis lung inflammation. JCI insight 1:e87168.

Choy EH and Panayi GS (2001) Cytokine pathways and joint inflammation in rheumatoid arthritis. $N$ Engl J Med 344:907-916.

Delmore JE, Issa GC, Lemieux ME, Rahl PB, Shi J, Jacobs HM, Kastritis E, Gilpatrick T, Paranal RM, Qi J, et al. (2011) BET bromodomain inhibition as a therapeutic strategy to target c-Myc. Cell 146:904-917.

Dienz O and Rincon M (2009) The effects of IL-6 on CD4 T cell responses. Clin Immunol 130:27-33.

Dinarello CA (2009) Immunological and inflammatory functions of the interleukin-1 family. Annu Rev Immunol 27:519-550.

Emerson MR, Gallagher RJ, Marquis JG, and LeVine SM (2009) Enhancing the ability of experimental autoimmune encephalomyelitis to serve as a more rigorous model of multiple sclerosis through refinement of the experimental design. Comp Med 59:112-128.

Filippakopoulos P, Picaud S, Mangos M, Keates T, Lambert JP, Barsyte-Lovejoy D, Felletar I, Volkmer R, Müller S, Pawson T, et al. (2012) Histone recognition and large-scale structural analysis of the human bromodomain family. Cell 149:214-231.

Filippakopoulos P, Qi J, Picaud S, Shen Y, Smith WB, Fedorov O, Morse EM, Keates T, Hickman TT, Felletar I, et al. (2010) Selective inhibition of BET bromodomains. Nature 468:1067-1073.

Furtado GC, Marcondes MC, Latkowski JA, Tsai J, Wensky A, and Lafaille JJ (2008) Swift entry of myelin-specific T lymphocytes into the central nervous system in spontaneous autoimmune encephalomyelitis. J Immunol 181:4648-4655.

Gacias M, Gerona-Navarro G, Plotnikov AN, Zhang G, Zeng L, Kaur J, Moy G, Rusinova E, Rodriguez Y, Matikainen B, et al. (2014) Selective chemical modulation of gene transcription favors oligodendrocyte lineage progression. Chem Biol 21:841-854.

Gilham D, Wasiak S, Tsujikawa LM, Halliday C, Norek K, Patel RG, Kulikowski E, Johansson J, Sweeney M, and Wong NC (2016) RVX-208, a BET-inhibitor for treating atherosclerotic cardiovascular disease, raises ApoA-I/HDL and represses pathways that contribute to cardiovascular disease. Atherosclerosis 247:48-57.

Hansen HC (2011) inventor, Resverlogix Corporation, assignee. Compounds for the prevention and treatment of cardiovascular diseases. U.S. patent $8,053,440$. 2011 Nov 8.

Hofstetter HH, Ibrahim SM, Koczan D, Kruse N, Weishaupt A, Toyka KV, and Gold R (2005) Therapeutic efficacy of IL-17 neutralization in murine experimental autoimmune encephalomyelitis. Cell Immunol 237:123-130.

Huang B, Yang XD, Zhou MM, Ozato K, and Chen LF (2009) Brd4 coactivates transcriptional activation of NF-kappaB via specific binding to acetylated RelA. Mol Cell Biol 29:1375-1387.

Inglis JJ, Criado G, Medghalchi M, Andrews M, Sandison A, Feldmann M, and Williams RO (2007) Collagen-induced arthritis in C57BL/6 mice is associated with a robust and sustained T-cell response to type II collagen. Arthritis Res Ther 9:R113.

Itzen F, Greifenberg AK, Bösken CA, and Geyer M (2014) Brd4 activates P-TEFb for RNA polymerase II CTD phosphorylation. Nucleic Acids Res 42:7577-7590.

Jahagirdar R, Zhang H, Azhar S, Tobin J, Attwell S, Yu R, Wu J, McLure KG, Hansen HC, Wagner GS, et al. (2014) A novel BET bromodomain inhibitor RVX-208, shows reduction of atherosclerosis in hyperlipidemic ApoE deficient mice. Atherosclerosis 236:91-100.

Kataoka H, Sugahara K, Shimano K, Teshima K, Koyama M, Fukunari A, and Chiba K (2005) FTY720, sphingosine 1-phosphate receptor modulator, ameliorates experimental autoimmune encephalomyelitis by inhibition of $\mathrm{T}$ cell infiltration. Cell Mol Immunol 2:439-448.
Khachigian LM (2006) Collagen antibody-induced arthritis. Nat Protoc 1:2512-2516. Kharenko OA, Gesner EM, Patel RG, Norek K, White A, Fontano E, Suto RK, Young PR, McLure KG, and Hansen HC (2016) RVX-297- a novel BD2 selective inhibitor of BET bromodomains. Biochem Biophys Res Commun 477:62-67.

Kolls JK and Lindén A (2004) Interleukin-17 family members and inflammation. Immunity 21:467-476.

Komiyama Y, Nakae S, Matsuki T, Nambu A, Ishigame H, Kakuta S, Sudo K, and Iwakura Y (2006) IL-17 plays an important role in the development of experimental autoimmune encephalomyelitis. J Immunol 177:566-573.

Kroenke MA, Carlson TJ, Andjelkovic AV, and Segal BM (2008) IL-12- and IL-23modulated T cells induce distinct types of EAE based on histology, CNS chemokine profile, and response to cytokine inhibition. J Exp Med 205:1535-1541.

Lefevre S, Meier FM, Neumann E, and Muller-Ladner U (2015) Role of synovial fibroblasts in rheumatoid arthritis. Curr Pharm Des 21:130-141.

Marusic S, Thakker P, Pelker JW, Stedman NL, Lee KL, McKew JC, Han L, Xu X Wolf SF, Borey AJ, et al. (2008) Blockade of cytosolic phospholipase A2 alpha prevents experimental autoimmune encephalomyelitis and diminishes development of Th1 and Th17 responses. J Neuroimmunol 204:29-37.

McCarthy KM, McDevit D, Andreucci A, Reeves R, and Nikolajczyk BS (2003) HMGA1 co-activates transcription in B cells through indirect association with DNA. J Biol Chem 278:42106-42114.

McLure KG, Gesner EM, Tsujikawa L, Kharenko OA, Attwell S, Campeau E, Wasiak S, Stein A, White A, Fontano E, et al. (2013) RVX-208, an inducer of ApoA-I in humans, is a BET bromodomain antagonist. PLoS One 8:e83190.

Mele DA, Salmeron A, Ghosh S, Huang HR, Bryant BM, and Lora JM (2013) BET bromodomain inhibition suppresses TH17-mediated pathology. J Exp Med 210:2181-2190.

Mertz JA, Conery AR, Bryant BM, Sandy P, Balasubramanian S, Mele DA, Bergeron L, and Sims RJ, III (2011) Targeting MYC dependence in cancer by inhibiting BET bromodomains. Proc Natl Acad Sci USA 108:16669-16674.

Nakae S, Nambu A, Sudo K, and Iwakura Y (2003) Suppression of immune induction of collagen-induced arthritis in IL-17-deficient mice. J Immunol 171:6173-6177.

Nicholls SJ, Puri R, Wolski K, Ballantyne CM, Barter PJ, Brewer HB, Kastelein JJ, Hu B, Uno K, Kataoka Y, et al. (2016) Effect of the BET protein inhibitor, RVX-208, on progression of coronary atherosclerosis: results of the phase $2 \mathrm{~b}$, randomized, double-blind, multicenter, ASSURE trial. Am J Cardiovasc Drugs 16:55-65.

Nicodeme E, Jeffrey KL, Schaefer U, Beinke S, Dewell S, Chung CW, Chandwani R, Marazzi I, Wilson P, Coste H, et al. (2010) Suppression of inflammation by a synthetic histone mimic. Nature 468:1119-1123.

Nikolic D, Rizzo M, Mikhailidis DP, Wong NC, and Banach M (2015) An evaluation of RVX-208 for the treatment of atherosclerosis. Expert Opin Investig Drugs 24: 1389-1398.

O'Connor RA, Prendergast CT, Sabatos CA, Lau CW, Leech MD, Wraith DC, and Anderton SM (2008) Cutting edge: th1 cells facilitate the entry of Th17 cells to the central nervous system during experimental autoimmune encephalomyelitis. J Immunol 181:3750-3754.

Phatnani HP and Greenleaf AL (2006) Phosphorylation and functions of the RNA polymerase II CTD. Genes Dev 20:2922-2936.

Picaud S, Wells C, Felletar I, Brotherton D, Martin S, Savitsky P, Diez-Dacal B, Philpott M, Bountra C, Lingard H, et al. (2013) RVX-208, an inhibitor of BET transcriptional regulators with selectivity for the second bromodomain. Proc Natl Acad Sci USA 110:19754-19759.

Plum SM, Park EJ, Strawn SJ, Moore EG, Sidor CF, and Fogler WE (2009) Disease modifying and antiangiogenic activity of 2-methoxyestradiol in a murine model of rheumatoid arthritis. BMC Musculoskelet Disord 10:46.

Schröder S, Cho S, Zeng L, Zhang Q, Kaehlcke K, Mak L, Lau J, Bisgrove D, Schnölzer M, Verdin E, et al. (2012) Two-pronged binding with bromodomaincontaining protein 4 liberates positive transcription elongation factor b from inactive ribonucleoprotein complexes. J Biol Chem 287:1090-1099.

Smale ST (2011) Hierarchies of NF-кB target-gene regulation. Nat Immunol 12:689-694.

Thakker P, Leach MW, Kuang W, Benoit SE, Leonard JP, and Marusic S (2007) IL-23 is critical in the induction but not in the effector phase of experimental autoimmune encephalomyelitis. J Immunol 178:2589-2598.

Toniolo PA, Liu S, Yeh JE, Moraes-Vieira PM, Walker SR, Vafaizadeh V, Barbuto JA, and Frank DA (2015) Inhibiting STAT5 by the BET bromodomain inhibitor JQ1 disrupts human dendritic cell maturation. J Immunol 194:3180-3190.

Tzartos JS, Friese MA, Craner MJ, Palace J, Newcombe J, Esiri MM, and Fugger L (2008) Interleukin-17 production in central nervous system-infiltrating T cells and glial cells is associated with active disease in multiple sclerosis. Am J Pathol 172: 146-155.

Wei G, Wei L, Zhu J, Zang C, Hu-Li J, Yao Z, Cui K, Kanno Y, Roh TY, Watford WT, et al. (2009) Global mapping of H3K4me3 and H3K27me3 reveals specificity and plasticity in lineage fate determination of differentiating CD4+ T cells. Immunity 30:155-167.

Williams RO (2004) Collagen-induced arthritis as a model for rheumatoid arthritis. Methods Mol Med 98:207-216.

Yang Z, Yik JH, Chen R, He N, Jang MK, Ozato K, and Zhou Q (2005) Recruitment of $\mathrm{P}-\mathrm{TEFb}$ for stimulation of transcriptional elongation by the bromodomain protein Brd4. Mol Cell 19:535-545.

Ye P, Rodriguez FH, Kanaly S, Stocking KL, Schurr J, Schwarzenberger P, Oliver P, Huang W, Zhang P, Zhang J, et al. (2001) Requirement of interleukin 17 receptor signaling for lung CXC chemokine and granulocyte colony-stimulating factor expression, neutrophil recruitment, and host defense. J Exp Med 194:519-527.

Zhang QG, Qian J, and Zhu YC (2015) Targeting bromodomain-containing protein 4 (BRD4) benefits rheumatoid arthritis. Immunol Lett 166:103-108.

Address correspondence to: Ravi Jahagirdar, Resverlogix Corp., Suite 300, 4820 Richard Road SW, Calgary, AB T3E 6L1, Canada. E-mail: ravi@ resverlogix.com 